\title{
The Importance of Natural Antioxidants in the Treatment of Spinal Cord Injury in Animal Models: An Overview
}

\author{
Angélica Coyoy-Salgado $\mathbb{D}^{1},{ }^{1}$ Julia J. Segura-Uribe $\mathbb{D}^{\circ},{ }^{2}$ Christian Guerra-Araiza ${ }^{(D)}{ }^{3}$ \\ Sandra Orozco-Suárez $\mathbb{D}^{2},{ }^{2}$ Hermelinda Salgado-Ceballos $\mathbb{D}^{2},{ }^{2}$ Iris A. Feria-Romero $\mathbb{D}^{2}{ }^{2}$ \\ Juan Manuel Gallardo $\left(\mathbb{D},{ }^{4}\right.$ and Carlos E. Orozco-Barrios ${ }^{1}{ }^{1}$
}

${ }^{1}$ CONACyT-Unidad de Investigación Médica en Enfermedades Neurológicas, Hospital de Especialidades Dr. Bernardo Sepúlveda, Centro Médico Nacional Siglo XXI, Instituto Mexicano del Seguro Social, Mexico City, Mexico

${ }^{2}$ Unidad de Investigación Médica en Enfermedades Neurológicas, Hospital de Especialidades Dr. Bernardo Sepúlveda, Centro Médico Nacional Siglo XXI, Instituto Mexicano del Seguro Social, Mexico City, Mexico

${ }^{3}$ Unidad de Investigación Médica en Farmacología, Hospital de Especialidades Dr. Bernardo Sepúlveda, Centro Médico Nacional Siglo XXI, Instituto Mexicano del Seguro Social, Mexico City, Mexico

${ }^{4}$ Unidad de Investigación Médica en Enfermedades Nefrológicas, Hospital de Especialidades Dr. Bernardo Sepúlveda, Centro Médico Nacional Siglo XXI, Instituto Mexicano del Seguro Social, Mexico City, Mexico

Correspondence should be addressed to Angélica Coyoy-Salgado; acoyoys@gmail.com and Carlos E. Orozco-Barrios; crls2878@gmail.com

Received 31 May 2019; Accepted 4 October 2019; Published 12 November 2019

Guest Editor: João C. M. Barreira

Copyright ( 2019 Angélica Coyoy-Salgado et al. This is an open access article distributed under the Creative Commons Attribution License, which permits unrestricted use, distribution, and reproduction in any medium, provided the original work is properly cited.

\begin{abstract}
Patients with spinal cord injury (SCI) face devastating health, social, and financial consequences, as well as their families and caregivers. Reducing the levels of reactive oxygen species (ROS) and oxidative stress are essential strategies for SCI treatment. Some compounds from traditional medicine could be useful to decrease ROS generated after SCI. This review is aimed at highlighting the importance of some natural compounds with antioxidant capacity used in traditional medicine to treat traumatic SCI. An electronic search of published articles describing animal models of SCI treated with natural compounds from traditional medicine was conducted using the following terms: Spinal Cord Injuries (MeSH terms) AND Models, Animal (MeSH terms) AND [Reactive Oxygen Species (MeSH terms) AND/OR Oxidative Stress (MeSH term)] AND Medicine, Traditional (MeSH terms). Articles reported from 2010 to 2018 were included. The results were further screened by title and abstract for studies performed in rats, mice, and nonhuman primates. The effects of these natural compounds are discussed, including their antioxidant, anti-inflammatory, and antiapoptotic properties. Moreover, the antioxidant properties of natural compounds were emphasized since oxidative stress has a fundamental role in the generation and progression of several pathologies of the nervous system. The use of these compounds diminishes toxic effects due to their high antioxidant capacity. These compounds have been tested in animal models with promising results; however, no clinical studies have been conducted in humans. Further research of these natural compounds is crucial to a better understanding of their effects in patients with SCI.
\end{abstract}

\section{Introduction}

Spinal cord injury (SCI) is a life-disrupting condition associated with high mortality and long-term morbidity, which may provoke severe consequences to patients, such as paraplegia or quadriplegia, and frequently continues as a terminal condition. According to the National SCI Statistical Center, an annual incidence of 17,500 new SCI cases is estimated. Since it is a frequent and severe motor injury, it becomes a potential economic, social, and family burden. In 2017, between 245,000 and 353,000 patients with SCI were alive in the United States, with an estimated lifetime cost of $\$ 1.6-\$ 4.8 \mathrm{M}$ per patient [1].

Among the leading causes of SCI are traffic and sports accidents, as well as falls and violence. Traumatic SCI 
occurrence shows a peak between the ages of 15 and 29 years and another peak over the age of 65 years, with an incidence rate of 3-4 times higher in males $[2,3]$. Depending on the severity of the lesion, patients show neurological deficits, which can range from loss of sensation to death, including paralysis, impaired bowel, bladder, and sexual function, as well as autonomic dysfunction [4-7].

Natural antioxidants are used as an alternative treatment for some neuropathologies, including SCI. This review is aimed at providing an overview of various natural compounds that produce beneficial effects for the treatment of SCI in animal models. Furthermore, the differences between animal responses to these various compounds are addressed to establish a better understanding of the cellular and molecular mechanisms occurring in the spinal cord following injury.

\section{Pathophysiology of SCI}

SCI can be divided into primary, secondary, and chronic phases $[8,9]$. The first phase is the result of the physical forces involved in the initial traumatic event, which commonly are the most critical elements of injury severity. These forces include compression, shearing, laceration, and acute stretch/distraction [10]. After the initial injury, a cascade of subsequent events is initiated. In this second phase, the injury expands and neurological deficits and outcomes are exacerbated $[11,12]$. Secondary SCI is a delayed and progressive injury following the first damage. Finally, a chronic phase, begins days to years after the injury, leading to neurological impairments in both orthograde and retrograde directions, including some brain regions $[13,14]$.

During the secondary cascade, some vascular changes are observed [15]. Furthermore, neutrophils and macrophages release superoxide anion and hydrogen peroxide as a means to sterilize the injury site. Infiltrating activated hematogenous phagocytic cells and tissue macrophages generate massive quantities of superoxide anion by nicotinamide adenine dinucleotide phosphate $(\mathrm{NADPH})$ oxidase as its primary source [16].

In addition, phagocytic inflammatory cells release reactive oxygen species (ROS). Free radicals react with polyunsaturated fatty acids leading to peroxidation and disruption of the typical phospholipid architecture of cellular and subcellular organelle membranes. Moreover, lipid peroxidation generates aldehyde products that impair the function of key metabolic enzymes, such as $\mathrm{Na}^{+} / \mathrm{K}^{+}$-ATPase [17].

SCI is generally characterized by an increase in cytokines, such as TNF- $\alpha$, IL- $1 \beta$, and IL- 6 that lead to upregulation of inflammatory and apoptotic agents, including NF- $\kappa \mathrm{B}, \mathrm{AP}-1$, JNK, p38 MAPK, and PGE2 [3].

After SCI, an upregulated liberation of excitatory amino acids, such as glutamate and aspartate, is observed due to the release from disrupted cells [18-20].

Finally, in the chronic phase, the glial scar, which is integrated by reactive astrocytes, microglia/macrophages, and extracellular matrix molecules-chondroitin sulfate proteoglycans in particular-prevents axon growth through it by acting as a physical barrier [21-25].
Consequently, it becomes necessary to develop reliable strategies and treatments for SCI patients. An essential strategy for SCI treatment is the reduction of ROS levels, which could be carried out using antioxidants or compounds that regulate ROS or modify their signaling pathways $[26,27]$.

\section{ROS Production and Spinal Cord Injury}

3.1. Oxidative and Nitrosative Stress. The homeostasis of redox mechanisms in the spinal cord is maintained in balance. However, under adverse conditions such as neurodegenerative diseases or traumas, this balance is altered. The group of biochemical and molecular reactions following SCI is called secondary damage. The most extensively studied and accepted mechanism of secondary damage is the injury produced by oxidative and nitrosative stress [28].

The essential molecule within oxidative stress is the superoxide $\left(\mathrm{O}_{2}{ }^{--}\right)$radical, which is produced by the reduction of an electron in an $\mathrm{O}_{2}$ molecule. This radical has ambivalent functions: it can act as an oxidizing or reducing agent. Despite being considered a modestly reactive free radical, it can react with other molecules to generate more reactive free radicals (Figure 1(a)). For example, the nitric oxide ( $\mathrm{NO}$ ) radicals produce peroxynitrite $\left(\mathrm{ONOO}^{-}\right)$, which has a higher oxidation potencial. Consecutively, the $\mathrm{ONOO}^{-}$radical can be protonated to form peroxynitrous acid $(\mathrm{ONOOH})$, which in turn can be decomposed into two highly reactive molecules, nitrogen oxide and hydroxyl radical $\left(\mathrm{NO}_{2}\right.$ and $\left.\mathrm{OH}\right)$. Furthermore, the $\mathrm{ONOO}^{-}$radical interacts with carbon dioxide $\left(\mathrm{CO}_{2}\right)$ as well, to produce nitrosoperoxycarbonate $\left(\mathrm{ONOOCO}_{2}\right)$, which decomposes into nitrogen oxide and carbonate $\left(\mathrm{CO}_{3}\right.$ ) radical (Figure 1(b)) [29].

Moreover, $\mathrm{O}_{2}{ }^{--}$can be dismuted by the superoxide dismutase (SOD) enzyme to form hydrogen peroxide $\left(\mathrm{H}_{2} \mathrm{O}_{2}\right)$ in the presence of $\mathrm{Fe}^{2+}$, which is later oxidized to $\mathrm{Fe}^{3+}, \mathrm{OH}$ and $\mathrm{OH}^{-}$(Fenton reaction). $\mathrm{Fe}^{3+}$ is released from its transporter and storage proteins (transferrin and ferritin, respectively) by $\mathrm{pH}$ acidification due to the traumatic impact (Figure 1(a)). Additionally, Fe is also released by the hemoglobin resulting from trauma [30].

3.2. Lipid Peroxidation. Lipid peroxidation (LP) is the oxidative degradation of unsaturated fatty acids, such as arachidonic acid, linoleic acid, eicosapentaenoic acid, and docosahexaenoic acid, by the action of oxygen-free radicals, which causes disruptions in the integrity of the cell membrane [31].

This process is carried out in three stages: initiation, propagation, and termination. The initiation phase occurs when a free radical ( $\mathrm{R}$ ) attacks and removes hydrogen along with its single electron from an allylic carbon of the fatty acid $(\mathrm{LH})$, generating an alkyl radical ( $\mathrm{L})$. The propagation phase starts with the participation of $\mathrm{O}_{2}$ to form the peroxyl radical (LOO). Subsequently, the radical LOO reacts with another fatty acid converting it into another alkyl radical $\mathrm{L}$ and $\mathrm{LOOH}$, propagating the oxidative state in a series of chain reactions leading to the destabilization of the membrane 


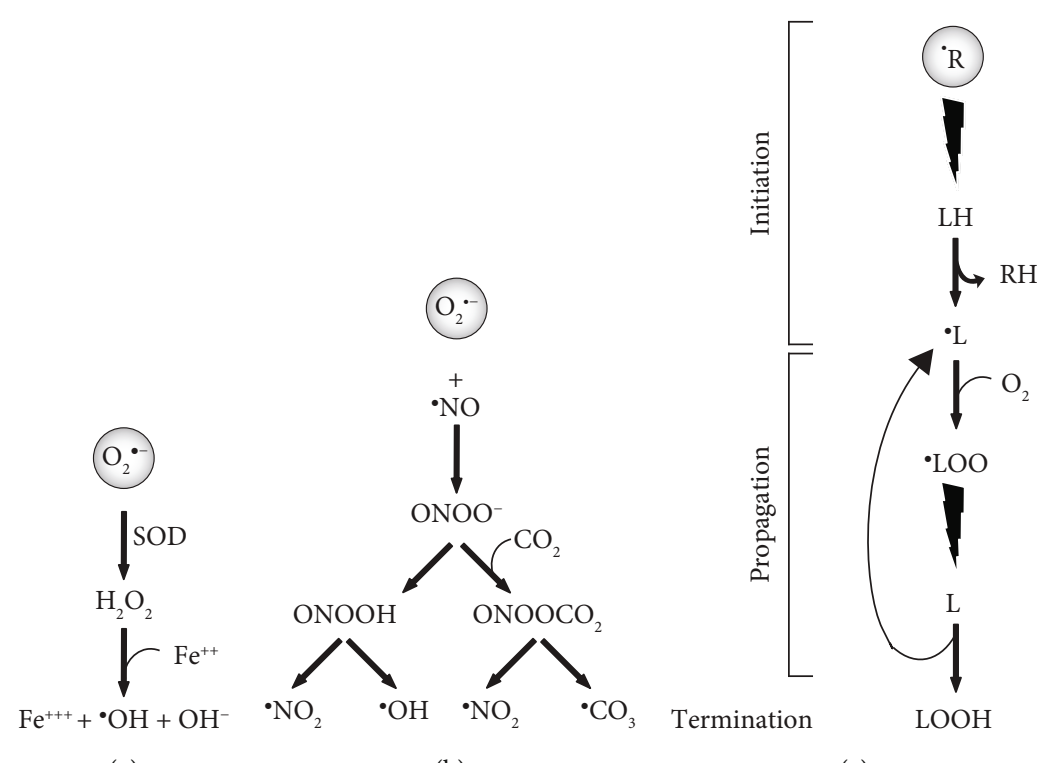

(a)

(b)

(c)

FIgURE 1: Main pathways of ROS and NOS in the central nervous system. (a) Fenton reaction; (b) peroxynitrite reaction; (c) lipid peroxidation. $\mathrm{O}_{2}{ }^{-}$: superoxide radical; SOD: superoxide dismutase; $\mathrm{H}_{2} \mathrm{O}_{2}$ : hydrogen peroxide; $\mathrm{Fe}^{++}$: ferrous iron; $\mathrm{Fe}^{+++}$: ferric iron; ${ }^{\circ} \mathrm{OH}$ : hydroxyl radical; $\mathrm{OH}$-: hydroxyl anion; NO: nitric oxide or nitrogen monoxide; $\mathrm{NO}_{2}$ : nitrogen dioxide; ONOO-: peroxynitrite anion; $\mathrm{CO}_{2}$ : carbon dioxide; $\mathrm{ONOOH}$ : peroxynitrous acid; $\mathrm{ONOOCO}_{2}$ : nitrosoperoxocarbonate; $\mathrm{CO}_{3}$ : carbonate radical; $\mathrm{R}$ : free radical; $\mathrm{RH}$ : neutralized radical; $\mathrm{LH}$ : polyunsaturated fatty acid; ' $\mathrm{L}$ : alkyl radical; $\mathrm{O}_{2}$ : molecular oxygen; LOO: peroxyl radical; LOOH: lipid hydroperoxide.

(Figure 1(c)). These reactions are terminated by the depletion of substrates, encountering another radical or a scavenger and ending with nonradical molecules. Within this group of reactions, a couple of toxic aldehyde products, 4hydroxynonenal (4-HNE) and 2-propenal (acrolein), are generated. The toxicity of 4-HNE and acrolein lies in their ability to bind to protein amino acids, altering their structure and function.

In contrast, another nontoxic product, malondialdehyde (MDA), together with the quantification of 4-HNE and acrolein, is widely used to measure LP levels. These markers have allowed the characterization of the LP in SCI models [32-34].

LP is an event that appears within the first 30 minutes after SCI and increases considerably from the first hour to a maximum point three hours after the SCI in a contusion model [35].

Another type of damage is caused by the NO radical which, as mentioned above, reacts with the radical $\mathrm{O}_{2}{ }^{--}$producing the $\mathrm{ONOO}^{-}$radical. This radical produces the nitration of proteins when interacting with the amino acid tyrosine, generating posttranslational modifications by converting tyrosine into 3-nitrotyrosine (3-NT). 3-NT is used as a biological marker of the action of $\mathrm{ONOO}^{-}$[36] and has been detected within the first hour after the injury and for several days. Nitric oxide synthase (NOS) in all its isoforms, including a mitochondrial variant, is responsible for the production of the radical NO. The expression of nNOS increases in the CNS after a traumatic injury [37].

\section{Effects of Natural Antioxidant Compounds on SCI}

Medicinal plants have been used for thousands of years. Herbs, roots, bulbs, and fruits contain different compounds which act as therapeutic ingredients [38]. Recently, traditional medicine has been the focus of attention in the treatment of some diseases, including SCI [39].

Due to their antioxidant characteristics and ROS modulation properties, many natural compounds could be useful to reduce ROS generated in the SCI (Table 1). For that reason, it is vital to study the mechanisms of action through which they perform their effects. Therefore, some examples of antioxidant compounds found in several plant species used as SCI treatment are described and discussed as follows.

\subsection{Extracts from Leaves}

4.1.1. Asiatic Acid. Traditional Chinese medicine has offered many proposals, including asiatic acid (AA) and other compounds. AA is extracted from the Chinese herb Centella asiatica. It is a pentacyclic triterpenoid compound with antiinflammatory, hepatoprotective, cardioprotective, neuroprotective, gastroprotective, and anticancer properties [40].

AA was proposed as SCI treatment for its therapeutic potential. Promising results were observed in the model used, in which Sprague-Dawley rats with induced SCI responded to the treatment: scores increased in the tests evaluated, Basso, Beattie, and Bresnahan (BBB) and inclined plane. Also, AA reduced myeloperoxidase activity, as well as interleukin-1 $\beta$ (IL-1 $\beta)$, interleukin-18 (IL-18), interleukin-6 (IL-6), tumor necrosis factor- $\alpha$ (TNF- $\alpha$ ), ROS, $\mathrm{H}_{2} \mathrm{O}_{2}$, and MDA levels. Moreover, the activity of superoxide dismutase (SOD) and the content of glutathione (GSH) increased with AA [41].

The mechanism of AA involves the activation of the nuclear factor- (erythroid-derived 2-) like-2 factor (Nrf2), a cytoprotective factor that regulates the expression of genes 


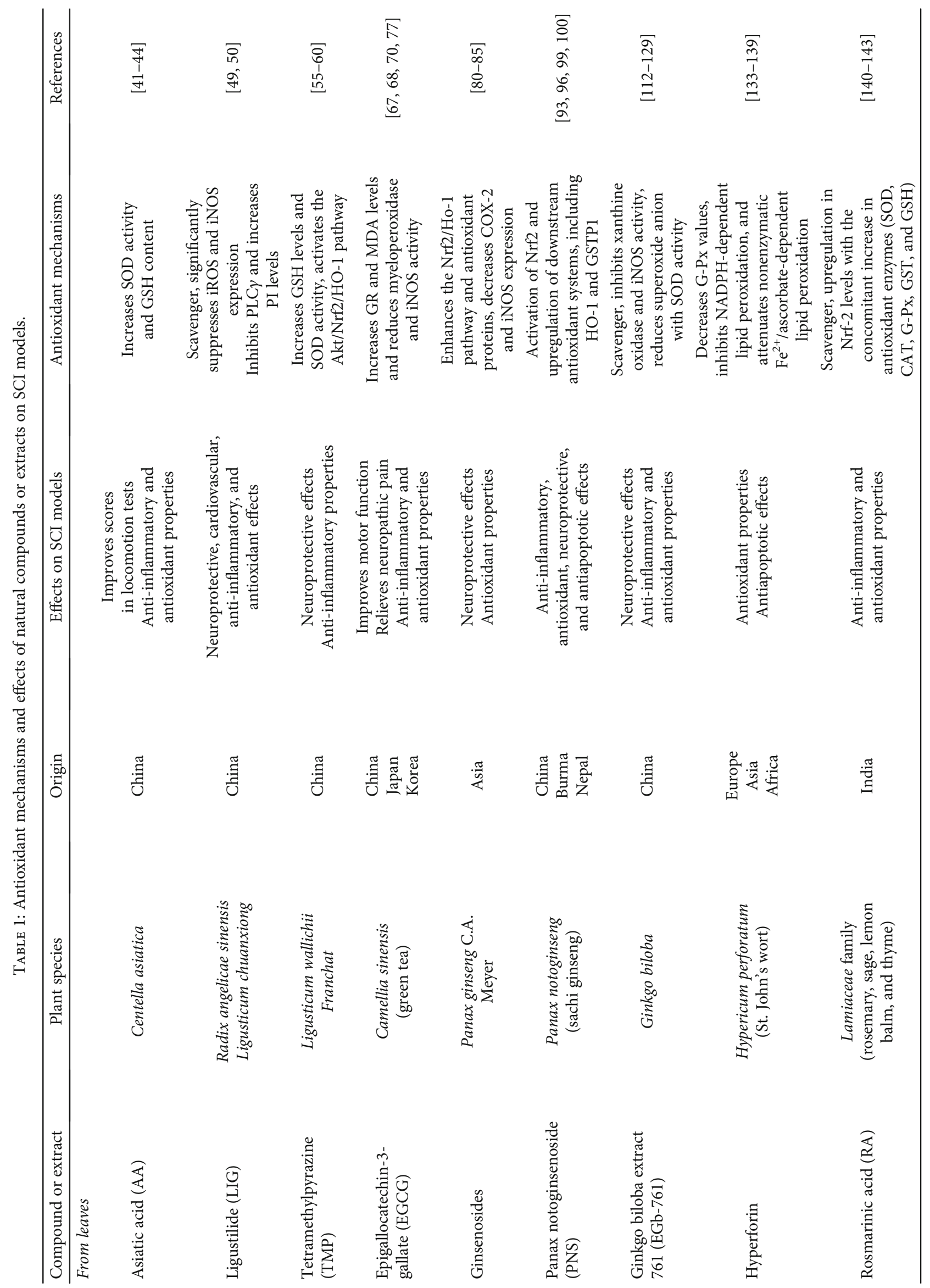




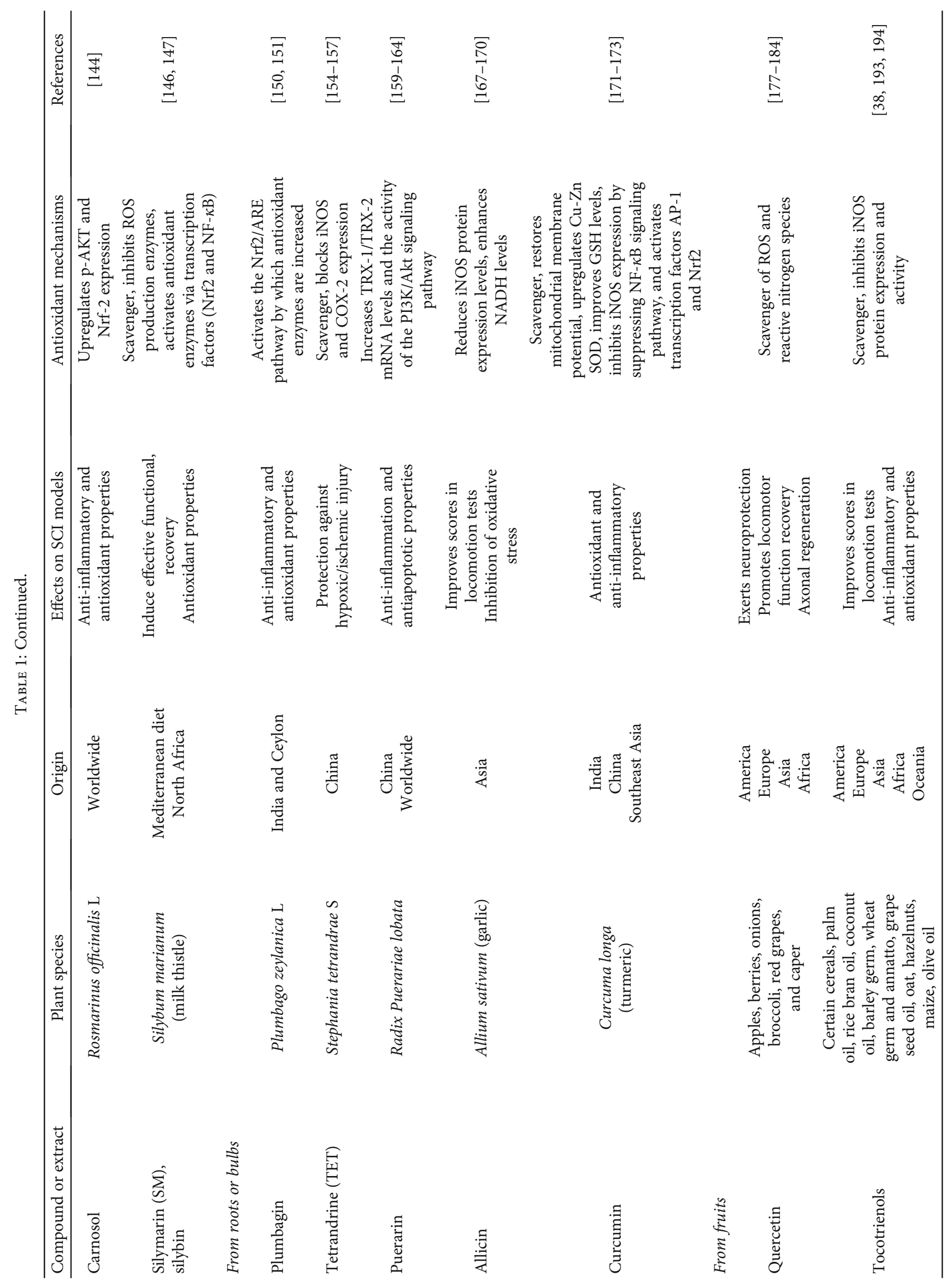




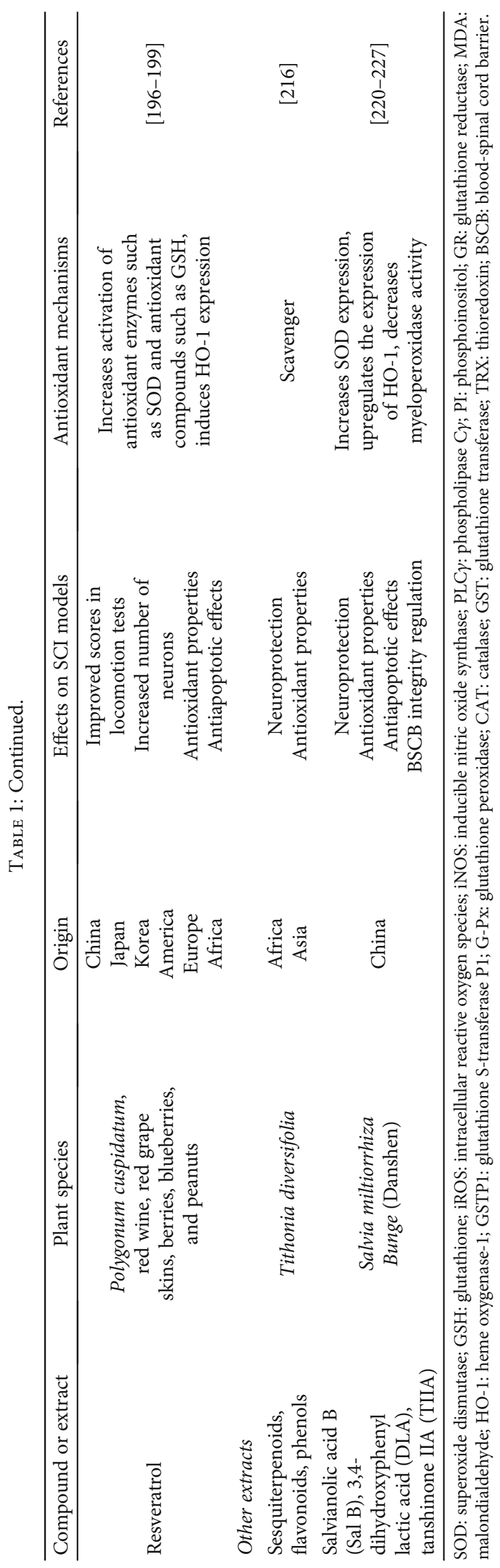


encoding antioxidant, anti-inflammatory, and detoxifying proteins, as well as heme oxygenase-1 (HO-1), a protein coded by a Nrf2-dependent gene, which degrades toxic heme groups; produces biliverdin, iron ions, and carbon monoxide; and contributes to angiogenesis [42]. The effects of AA can also be attributed to the inhibition of ROS and the nucleotide-binding domain and leucine-rich repeat- (LRR-) containing (NLR) family pyrin domain-containing 3 (NLRP3) inflammasome pathway. The NLRP3 inflammasome is a multiprotein complex that activates caspase-1, leading to the secretion of proinflammatory molecules such as IL- $1 \beta$ and IL-18. Under normal conditions, NLRP3 remains autorepressed, but this changes with increasing concentrations of damage-associated and pathogenassociated molecular patterns as well as ROS, which are crucial elements for NLRP3 activation [43].

SCI can lead to secondary acute lung injury (ALI). AA administration significantly attenuates pulmonary permeability index and pulmonary histologic conditions and exhibits a protective effect on SCI-induced ALI by alleviating the inflammatory response through inhibiting NLRP3 inflammasome activation and oxidative stress with the upregulation of Nrf2 protein levels [44]. The use of AA may be a potential efficient therapeutic strategy for the treatment of SCI and SCI-induced ALI $[41,44]$.

4.1.2. Ligustilide. Ligustilide (3-butylidene-4,5-dihydrophthalide) is the main lipophilic constituent of the Umbelliferae family of medicinal plants, including Radix angelicae sinensis and Ligusticum chuanxiong [45]. As it crosses the blood-brain barrier, ligustilide (LIG) exerts marked neuroprotective effects against several CNS pathologies, including forebrain ischemic injury in mice, permanent forebrain ischemia and focal cerebral ischemia/reperfusion in rats [46-48]. In addition, LIG exhibited a wide range of pharmacologic effects in vitro and in vivo: cardioprotective, antioxidant, anti-inflammatory, and neuroprotective activities [49]. Xiao et al. demonstrated that LIG promotes functional recovery in rats with SCI by preventing the production of ROS. Treatment with LIG significantly increased $\mathrm{BBB}$ scores and reduced the time for recovery of coordination in rats with SCI. Furthermore, LIG suppressed SCI-induced production of ROS, inducible nitric oxide synthase (iNOS), inflammation, and JNK signaling. However, further studies are needed to identify the mechanisms by which LIG regulates neuroprotection and mediates locomotor recovery following SCI [50].

4.1.3. Tetramethylpyrazine. Tetramethylpyrazine (TMP), an alkaloid extracted from the Chinese medicinal herb Ligusticum wallichii Franchat (chuanxiong), is widely used in the treatment of ischemic stroke and cardiovascular disease [51-53] and has shown anti-inflammatory and neuroprotective effects against SCI as well $[54,55]$. In different models of SCI, TMP improved locomotor functions when compared with control animals [52, 56-60]. This improvement in motor activity correlated positively with a decreased area of the injury-induced lesion and increased tissue sparing [51, 58, 59]. Furthermore, a decreased permeability of the blood-spinal cord barrier was also observed [55]. TMP promoted angiogenesis increasing vessel number, vessel volume fraction, and connectivity as well $[58,60]$. A mechanism proposed for these effects is the overexpression of PGC-1, a transcriptional coactivator linked to energy metabolism in the mitochondria. This protein is involved in a variety of neurological disorders and apoptosis [57]. Additionally, TMP prevents the reduction of HO1 and Akt phosphorylation produced in SCI [55].

Regarding neuropathic pain produced in SCI, TMP treatment increased both mechanical withdrawal thresholds and thermal withdrawal latencies $[53,61]$. The effect of TMP on neuropathic pain relies on neuronal survival in the dorsal horn and the inhibition of astrocyte activation [62]. In the case of neuronal survival, TMP can modulate mediators of apoptosis such as Bcl-2 and caspase-3 [61], whereas, in the case of the inhibition of astrocyte activation, TMP releases matrix metalloproteinase-2/9 (MMP-2/9) to induce central sensitization and maintain neuropathic pain [62]. Moreover, TMP treatment decreased the expression of pSTAT3. Therefore, TMP could attenuate neuropathic pain by the inhibition of the JAK/STAT3 pathway [53].

TMP shows anti-inflammatory effects in SCI: TMP treatment reduced the expression of proinflammatory cytokines TNF- $\alpha$, IL- $1 \beta$, macrophage migration inhibitory factor positive (MIF), IL-18, IL-2, and COX-2 [51-56]; upregulated the expression of anti-inflammatory cytokines IL-10, I- $\kappa \mathrm{B}$, and IL10; inhibited the activation of NF- $\kappa \mathrm{B}$ [51, 52]; alleviated neutrophil infiltration; and attenuated microglia activation [51, 52, 54]. Matrix metalloproteinases 2 (MMP2) and 9 (MMP9) are implicated in neuropathic pain by mediating inflammatory pathways. TMP administration induces downregulation of both metalloproteases [60]. Thus, TMP prevents inflammation in spinal cord injury in rats.

TMP reduced neuronal apoptosis by increasing Bcl-2 and reducing Bax, as well as reduced TUNEL-positive cells and caspase- 3 and caspase- 9 activities [55, 57, 59-61]. Furthermore, TMP increases miR-21 expression, thus decreasing the expression of its targets FasL, PDCD4, and PTEN [59]. In addition to miR-21, TMP decreased the expression of miR-214-3p by increasing the expression of Bcl2L2, suggesting that TMP can modulate apoptosis in SCI [63].

Finally, TMP can decrease ROS in SCI. In rats, TMP treatment decreased lipid peroxidation and increased glutathione levels and superoxide dismutase activity. Also, TMP regulated the expression of Nrf2 mRNA and its binding in HO-1 promoter positively. Thus, TMP showed effects against ROS through the activation of the Akt/Nrf2/HO1 pathway $[55,56]$.

4.1.4. Epigallocatechin-3-Gallate. Epigallocatechin-3-gallate (EGCG) is the most abundant polyphenol found in green tea, for which multiple benefits have been described: anticholesterolemic, antioxidant, and anti-inflammatory functions, as well as a modulator of apoptosis. In the CNS, a neuroprotective effect has been shown in a wide range of neurodegenerative diseases in various animal models. In the case of SCI, the administration of EGCG improves both motor and sensory (allodynia, nociception, and hyperalgesia) functions in 
acute and chronic models. EGCG administration $(100 \mathrm{mg} / \mathrm{kg}$ weight) produces the recovery of motor function [64-68] accompanied by a decrease of the injury area and an increase in the number of neurons [64]. The main mechanisms underlying the effects of EGCG range from the induction of the expression of neurotrophic factors, such as BDNF, GDNF $[66,69]$, and NT3, and their receptors, Trk-B, Trk-C, and NGFR-p75 [69], to the expression of growth factors, such as FGF2 and VEGF [70], accompanied by an increase of GAP43 [64], and the attenuation of myelin degradation $[65,66]$. In vitro models have shown that EGCG decreases the inhibitory activity of neuritic growth and the collapse of the growth cone induced by NOGO-66. This effect was observed through the $67 \mathrm{kDa}$ laminin receptor to which EGCG binds with high affinity [71]. Also, EGCG attenuates axon repulsion mediated by semaphorin [72]. Evidence shows that EGCG has protective effects for the modulation of neurotrophic factors and their receptors, as well as axonal sprouting. EGCG relieves the neuropathic pain produced in SCI and constriction of the sciatic nerve models [67, 68,73-75], allowing the recovery of sensory functions by improving tactile allodynia and mechanical nociception. EGCG also increases the latency of paw withdrawal and tail-flick tests $[67,68]$. Within the molecular mechanisms of EGCG to alleviate neuropathic pain is the reduction of the expression of CX3CL1, a fractalkine chemokine that has been shown to play an essential role in the development of neuropathic pain. The administration of EGCG reduced thermal hyperalgesia, as an effect of the reduction of CX3CL1 protein expression but not its RNA. Therefore, EGCG is suggested to act as a mediator of nociceptive signaling between neurons and glial cells [75].

Another mechanism that has been studied is the suppression of TLR4 expression. Several studies have demonstrated the involvement of TLRs and inflammation in the development of neuropathic pain. Although EGCG can inhibit other effector molecules of inflammation, the sole inhibition of TLR4 can inhibit the TLR4/NF- $\kappa$ B pathway. Also, EGCG induces the decrease of HMGB1, which has been implicated in chronic neuropathic pain by joining to TLR4 and activating the immune response [73].

EGCG has a more significant action on neuropathic pain than on motor recovery. In the short term, the administration of EGCG only affects sensory recovery but not on motor recovery [76].

EGCG can inhibit the expression of RhoA, FASN, and TNF- $\alpha$. In addition to limiting axonal regeneration, Álvarez-Pérez et al. demonstrated that RhoA participates in the generation of neuropathic pain. In the case of FASN, which is an enzyme that synthesizes palmitate, a lipid that is capable of activating the synthesis and release of proinflammatory agents, EGCG induces the decrease of FASN and the activation of the inflammatory pathways involved in neuropathic pain [74].

In addition to the mediators of inflammation described in neuropathic pain and a potent anti-inflammatory effect, EGCG is able to attenuate the activity of myeloperoxidase and attenuate the expression of inflammatory cytokines, such as TNF- $\alpha$, IL- $1 \beta$, IL-6, IL-2, MIP1, RANTES, nitrotyrosine, iNOs, COX, PARP, NF- $\kappa$ B, and HMGB1 $[65,73]$, accompanied by an increase in the anti-inflammatory cytokine IL-10 as well [73].

EGCG also modified the expression of IL-4, IL-12p70, and TNF- $\alpha 1$. The mechanism depends on the nuclear translocation of the p65 subunit of NF- $\kappa \mathrm{B}$, consequently inhibiting its activity along with the inflammatory pathways that it regulates. EGCG can modulate the expression of macrophages type M1 and M2. Both macrophage populations have different actions in the mechanisms of inflammation, allowing opposed states [70]. Therefore, EGCG modulates the expression of inflammatory cytokines that affect the expression and activity of its inductors.

EGCG also has antioxidant effects. In SCI, EGCG significantly reduces MDA levels [64] and increases glutathione reductase. This overexpression is accompanied by the decrease of isoprostanes in urine and the suppression of HO-1 [77].

As survival mechanisms, on the one hand, EGCG increases the expression of Bcl-2 and survivin and, on the other hand, it decreases the expression of Bax [64-66, 68]. These effects are reflected by the TUNEL assay low positivity in treated spinal cords [64]. Furthermore, PARP is a protein that is capable of inducing death by the depletion of NAD and ATP. EGCG decreases the expression of PARP, which could contribute to the reduction of Bax and the increase of $\mathrm{Bcl} 2$ [65].

4.1.5. Ginsenosides. Ginsenosides are steroid-like molecules which have a four trans-ring structure with sugar residues attached [78]. Ginsenosides Rb1, Rg1, and $\mathrm{Rg} 3$ show multiple pharmacological activities on the cardiovascular and immune systems, as well as neuroprotective effects [79, 80].

Ginsenosides can act as antioxidants or scavengers for free radicals [81], increase the activity of superoxide dismutase, and reduce lipid peroxidation $[82,83]$.

$\mathrm{Rb} 1$ protects neurons against oxidative injury, enhancing the Nrf2/HO-1 pathway since the activation of Nrf2 upregulates the transcription of multiple antioxidant response element-controlled genes [84, 85]. Also, ginsenoside Rb1 protects the ischemic brain through upregulation of the expression of Bcl-xL in vitro and in vivo [86].

Panax ginseng C.A. Meyer ( $P$. ginseng) is an herb commonly known as Asian or Korean ginseng [87], which contains ginsenoside saponins that account for the pharmacological efficacy [88].

$P$. ginseng has 150 different types of ginsenoside saponins, but Rb1, Rb2, Rc, Rd, Re, and Rg1 constitute more than $90 \%$ of the total ginsenosides [89].

Regarding the effects on SCI, the ginsenosides Rb1 and Rg1, extracted from Panax ginseng C.A. Meyer, were efficient neuroprotective agents for spinal cord neurons in vitro survival assays. These compounds protected spinal neurons from excitotoxicity induced by glutamate and kainic acid, as well as oxidative stress induced by $\mathrm{H}_{2} \mathrm{O}_{2}$. The neuroprotective effects are dose dependent, which optimal doses were 20-40 $\mathrm{mM}$ for both Rb1 and Rg1 [81]. 
Other authors demonstrated in vitro that dgRb1 (dihydroginsenoside $\mathrm{Rb} 1$ ), a stable chemical derivative of gRb1, upregulated VEGF and Bcl-xL expression and facilitated neuronal survival through the hypoxia response element (HRE) and signal transducers and activators of transcription 5 (Stat5) response element [90]. Consistently, Sakanaka et al. showed that an intravenous infusion of dgRb1 improved SCI in rats, as well as ischemic brain damage in MCA-occluded rats. The dgRb1-treated groups showed significant improvement of motor activity and behavioral abnormalities concerning locomotor and rearing activities and BBB score in a dose-dependent manner after SCI [90].

Kim et al. reported that ginseng extracts injected intraperitoneally improved recovery after contusive SCI in rats [91]. Additionally, Zhu et al. showed that the oral administration of red ginseng extract promoted the recovery from the motor and behavioral abnormalities in rats with SCI. Furthermore, this extract also stimulated neuronal restoration in the injured spinal cord by inhibiting the inflammatory processes and upregulating the expression of neuroprotective factors (VEGF and Bcl-xL) [92].

Wang et al. showed that ginseng treatment significantly downregulated oxidative stress on spinal injury in rats by enhancing antioxidant proteins and decreasing inflammatory proteins and proinflammatory cytokines [93].

Moreover, different doses of $P$. ginseng showed a significant improvement in locomotor function after spinal injury in rats. P. ginseng treatments decreased the expression of COX-2 and iNOS at the lesion site and the cavity area. These results suggest that $P$. ginseng may improve the recovery of motor function after SCI [91].

4.1.6. Panax Notoginsenoside. Unlike many other herbal medicines with a highly variable range of applications, Panax notoginseng, which is classified as a warm, sweet, slightly bitter, and nontoxic in Chinese medicine, has protective actions against cardiovascular diseases and diabetes [94, 95]. Moreover, many pharmacological activities of $P$. notoginseng, such as antioxidant, anti-inflammatory, hypoglycemic, antihyperlipidemic, anticoagulation, neuroprotective, and hepatoprotective effects, as well as antitumor and estrogen-like activities, have been reviewed [96].

Over 200 chemical constituents, including saponins, flavonoids, phytosterols, saccharides, polysaccharides, amino acids, fatty acids, dencichine, cyclopeptides, volatile oils, aliphatic acetylene hydrocarbons, and trace elements, have been isolated from $P$. notoginseng [97].

Panax notoginsenoside (PNS) is the principal active ingredient extracted from $P$. notoginseng, which main components are ginsenoside Rb1 (29.86\%), Rg1 (20.46\%), Rd (7.96\%), Re (6.83\%), and notoginsenoside R1 (2.74\%) [98]. PNS shows many beneficial effects, including anti-inflammation, antiedema, antioxidation, and antiapoptosis $[96,99]$, as well as neuroprotection in animal models of cerebral ischemia/reperfusion injury [100].

Compelling neuroprotective effects of PNS were demonstrated in a spinal cord ischemia-reperfusion injury model. SCI was induced in rats previously treated with PNS, in which the BBB scores significantly increased, as well as the number of neurons and a restored neuronal morphology observed by a histopathological examination. Furthermore, PNS decreased cytokine levels, as well as the expression of AQP-4 after the injury, suggesting an antiedema effect. An antiapoptotic mechanism of PNS was also verified since the treatment reduced the expression of Fas and FasL and inhibited injury-induced apoptosis [101].

4.1.7. Ginkgo biloba Extract 761 (EGb-761). Ginkgo biloba (Ginkgoaceae) is an ancient Chinese tree which has been cultivated and held sacred for its health-promoting properties [102]. EGb-761 is a patented extract from the leaves of the Ginkgo biloba tree composed of flavonoids (24\%), ginkgolide (3.1\%), bilobalides (2.9\%), and organic acids (5-10\%), particularly a ginkgo glycoside [103].

As one of the EGb-761 major constituents, ginkgolide B can improve hemorrhage, edema, necrosis, and inflammatory cell infiltrates in the injured spinal cord [39].

EGb-761 decreases blood viscosity, thereby increasing microcirculation [104], and modifies neurotransmission [105] and neuroplasticity as well [106]. Ginkgo biloba extracts have been used for the treatment of diseases related to the CNS, including brain injury, neurodegenerative disorders, and degenerative dementia [107-109].

EGb-761 also enhances cognition, reduces the detrimental effects of ischemia [110], shows neuroprotective effects, and enhances neurogenesis after ischemic stroke [111].

EGb-761 possesses antioxidant and free radicalscavenging activities $[112,113]$. In neurons treated with hydrogen peroxide, EGb-761 reduced oxidative stress and increased the viability of neurons [114, 115]. EGb-761 inhibits xanthine oxidase activity [116], reduces the production of superoxide anion, and inhibits SOD in human postmortem brain tissue [117]. By using electron spin resonance spectrometry, it was demonstrated that EGb-761 is a potent superoxide anion scavenger with SOD activity [118]. Also, EGb-761 can scavenge peroxyl radicals [119].

In spinal cord ischemic injury, EGb-761 protected spinal cord neurons in vivo, as well as hydrogen peroxideinduced spinal cord neuronal death in vitro [120]. During the acute phase after SCI, EGb-761 administration significantly reduced secondary injury-induced tissue necrosis and cell apoptosis and improved functional performance in rats [121].

EGb-761 performs its neuroprotective effects through scavenging free radicals, lowering oxidative stress, reducing neural damage, and preventing platelet aggregation, as well as its anti-inflammatory properties [122-124].

EGb-761 protects against ischemic SCI via their antioxidant effects in a rat model [125]. Furthermore, EGb-761 decreases SOD downregulation and significantly reduces MDA levels in spinal cord ischemia/reperfusion in rabbits $[126,127]$. In another study, EGb-761 was not able to demonstrate a uniform effect on the biochemical markers of spinal cord ischemia/reperfusion in rats. However, histopathologic data appear to show a protective effect of EGb-761 on the spinal cord tissue [128].

Research has demonstrated that iNOS expression is upregulated after SCI. In contrast, EGb761 can suppress 
iNOS expression and prevent neuronal death in SCI rats: in the treated group, the area of cavities was smaller, and the demyelinated zones were limited at and around the site of the SCI in comparison to the control group [129].

In acute spinal cord contusion in rats, cell apoptosis increased until day 14 after the injury. Furthermore, seven days after the injury, the number of apoptotic cells significantly decreased in the EGb761-treated group [121].

\subsubsection{Hyperforin}

Hypericum perforatum, also known as St. John's wort, hypericum, or millepertuis, is a member of the family Hypericaceae. Native from Europe, western Asia, and northern Africa, this herbaceous perennial plant currently can be found worldwide. The crude drug, known as Hyperici herba, is collected from the upper part of the aerial parts of the plant before or during the flowering period [130-132].

$H$. perforatum has demonstrated neuroprotective activities. Neuroprotection can be achieved by a direct action on one or several mechanisms, such as an antiapoptotic effect, or indirectly, through antioxidant properties. Chemically, structure-activity relationships suggest that a sugar side chain of flavonoids might be essential for neuroprotective activities [133] and multiple hydroxyl groups confer substantial antioxidant properties [134].

The active component of $H$. perforatum is hyperforin $[135,136]$, which reduces the overload of $\left[\mathrm{Ca}^{2+}\right]$ through NMDA receptor modulation in neurons $[136,137]$. H. perforatum standardized extract protects against enzymatic and nonenzymatic lipid peroxidation of rat brain, inhibiting $\mathrm{NADPH}$-dependent lipid peroxidation and attenuating nonenzymatic $\mathrm{Fe}^{2+} /$ ascorbate-dependent lipid peroxidation in cerebral cortex mitochondria [138].

After SCI in rats, $H$. perforatum showed a reduction in oxidative stress, apoptosis, and intracellular $\mathrm{Ca}^{2+}$ influx values through the regulation of the TRPM2 (transient receptor potential melastatin 2) and TRPV1 (transient receptor potential vanilloid 1) channels in dorsal root ganglion (DRG) neurons. Additionally, H. perforatum induced protective effects on lipid peroxidation and decreased GSH-Px values in the DRG neurons [139].

\subsubsection{Rosmarinic Acid and Carnosol. Rosmarinic acid (RA) is} a polyphenol found in the Lamiaceae family, abundantly present in rosemary, sage, lemon balm, and thyme. RA is a natural antioxidant with free radical scavenging and potential biological effects against oxidative stress and inflammation [140-142].

Shang et al. showed that RA treatment significantly decreased oxidative stress and enhanced the antioxidant status in post-SCI rats. Treatment with RA regulated redox homeostasis and oxidative stress markers, such as ROS, lipid peroxides, and sulfhydryl and carbonyl groups in proteins. RA treatment also caused the upregulation in Nrf-2 levels with the concomitant increase in antioxidant enzymes, such as SOD, CAT, GPx, GST, and GSH, and exerted antiinflammatory effects through the downregulation of NF- $\kappa$ B and proinflammatory cytokine levels (IL-6, IL- $1 \beta$, TNF- $\alpha$, and MCP-1) after SCI [143].

Carnosol, an orthodiphenolic diterpene with excellent antioxidant potential, is also found in rosemary. Wang et al. showed the protective role of carnosol against SCI-induced oxidative stress and inflammation through modulating NF- $\kappa$ B, COX-2, and Nrf-2 levels in Wistar rats. After the significant increase in ROS generation, oxidant levels, lipid peroxide content, protein carbonyl and sulfhydryl levels, and the reduction of the antioxidant status generated by induced SCI, carnosol treatment regulated inflammation key proteins and redox status through the significant downregulation of $\mathrm{NF}-\kappa \mathrm{B}$ and COX-2 levels and the upregulation of p-AKT and Nrf-2 expression [144].

4.1.10. Silymarin and Silybin. Silymarin (SM) is a mixture of flavonoids extracted from Silybum marianum (milk thistle) plant, including flavonolignans (silybin A and silybin B, isosilybin A, isosilybin B, silychristin, and silydianin), as well as fatty acids and polyphenols [145]. SM can contribute to antioxidant defenses as a scavenger of free radicals and by inhibiting specific free radical production enzymes. It also maintains an appropriate redox status by activating some enzymes and nonenzymatic antioxidants via transcription factors, including Nrf2 and NF- $\kappa$ B. Furthermore, SM activates the synthesis of protective molecules, such as thioredoxin (Trx), heat shock proteins, and sirtuins [146].

SM and silybin inhibited cell proliferation in cultures of glial cells in a concentration-dependent manner, as well as mixed cortical and spinal neuronal/glial cells against peroxide toxicity, and protected spinal cord neuronal/glial, glial and microglial cell cultures from LPS stimulation or peroxide toxicity. SM and silybin attenuated peroxide-induced ROS formation, with SM being more effective than silybin. Moreover, intrathecal administration of SM immediately after SCI effectively improved hindlimb locomotor behavior in the rats. These findings showed that SM and silybin exhibit general neuroprotective actions in the CNS [147].

Silybin elicits neuroprotection by the inhibition of peroxide-induced ROS through neuroinflammation and activation of glial cells, by modulating NF- $\kappa$ B or protein kinase $\mathrm{C}$ (PKC), as well as apoptosis, through inhibiting p53 and caspase-9, among other signaling pathways [147, 148].

\subsection{Extracts from Roots or Bulbs}

4.2.1. Plumbagin. Plumbagin, an analog of vitamin K3 isolated from the root of Plumbago zeylanica L, activates the Nrf2/ARE pathway resulting in the upregulation of target genes and increased resistance to oxidative and metabolic insults of neurons in culture and to ischemic stroke in vivo [149]. In a rat model, post-SCI treatment with plumbagin reduced SCI-induced oxidative stress and proinflammatory mediators [150]. SCI decreased the antioxidant levels of both nonenzymatic (GSH) and enzymatic antioxidants (NQO1, GST, GPx, SOD, and CAT) in sham rats. However, a significant increase in the antioxidant pool was observed in SCI rats treated with plumbagin. 
Moreover, it is well-known that Nrf2 activates the antioxidant machinery of cells [151]. Interestingly, plumbagin showed a significant upregulation of Nrf-2 in SCI, which suggests an essential role of plumbagin in cytoprotection as a potent Nrf-2 inducer [150].

4.2.2. Tetrandrine. Tetrandrine (TET), a bisbenzylisoquinoline alkaloid extracted from the roots of the Chinese medicinal herb Stephania tetrandrae S Moore, is a potential therapeutic candidate against cancer [152, 153], inflammation [154], and brain ischemia/reperfusion injury [155].

TET is a calcium channel blocker that can protect the liver, heart, small bowel, and brain from ischemia/reperfusion injury by inhibiting damaging factors, such as lipid peroxidation, generation of reactive oxygen species, production of cytokines and inflammatory mediators, neutrophil recruitment, and platelet aggregation [156].

Bao et al. studied the protective effect of TET on rat spinal cord astrocytes with oxygen-glucose-serum deprivation/reoxygenation-induced injury [157]. As expected, this intentional insult which mimics hypoxic/ischemic conditions in vivo caused considerable oxidative stress: an increase in ROS and MDA content, as well as a decreased SOD activity. Also, it increased the expression of proapoptotic Bax and caspase- 3 proteins, as well as the reduction of the antiapoptotic protein Bcl-2 [158]. The results of TET as a pretreatment to oxygen-glucose-serum deprivation/reoxygenation injury showed a dose-dependent suppression of Akt phosphorylation and NF- $\kappa \mathrm{B}$ activity and inhibition of the elevated caspase-3 activity. Additionally, TNF- $\alpha$, IL- $1 \beta$, and IL-6 accumulation induced by hypoxic/ischemic conditions were diminished. Overall, these results show a protective effect of TET against hypoxic/ischemic injury in spinal cord astrocytes through the $\mathrm{PI} 3 \mathrm{~K} / \mathrm{AKT} / \mathrm{NF}-\kappa \mathrm{B}$ signaling pathway attributable to its antioxidant and anti-inflammatory properties [157].

4.2.3. Puerarin. Puerarin, a natural isoflavone, is the main constituent of Radix Puerariae lobata. In SCI, puerarin has shown neuroprotective effects by improving motor function, mainly in ischemia-reperfusion [159-161] as well as in traumatic injury models [162]. Some mechanisms of neuroprotection have been described. As SCI causes the elevation of glutamate levels and mGluRs mRNA expression, which lead to neuronal injury, it has been shown that puerarin administration decreases both the excessive delivery of glutamate and the activation of mGluRs [160]. Also, puerarin upregulates the expression of GAP43, promoting the regeneration of nerve fibers [163]. Another mechanism of protection by puerarin is the inhibition of Cdk5 and p25. Cdk25 causes neuronal death and often is accompanied by the accumulation of p35 and p25, which in turn activates Cdk5, inducing feedback for the stimulus of the injury [161].

Moreover, puerarin can attenuate histological damage, decrease neuron death, and inhibit glial cell activation. These effects can be promoted by increasing the activity of the PI3K/Akt signaling pathway, which is involved in axonal outgrowth and the promotion of antioxidant and antiapoptosis effects [162].

Puerarin diminishes neuroinflammation $[162,164]$ by decreasing the activity of $\mathrm{NF}-\kappa \mathrm{B}$ and proinflammatory cytokines, such as IL-6, IL- $1 \beta$, and TNF- $\alpha$ [164]. Regarding apoptosis, puerarin reduces ROS by increasing thioredoxin- (TRX-) 1/TRX-2 mRNA levels, which are known to regulate apoptosis by modulating the redox ratio of the cell [159].

4.2.4. Allicin. Allium sativum (garlic) is a common and tasty ingredient found all over the world, which also has been used for medicinal purposes. In ancient Chinese and Indian medicine, it was used to treat several conditions, including leprosy, infections, and snake bites. Throughout history, garlic has been used to treat cardiovascular diseases and reduce high blood glucose concentration, blood pressure, and cholesterol levels. More recently, its antitumor, antiinflammatory, antifungal, and antimicrobial effects have been studied [165, 166].

Garlic contains many substances, from which allicin is the principal chemical component responsible for its biological activity [167].

Allicin is formed during the chopping, crushing, or chewing of garlic cloves through a chemical interaction between alliin, a sulfur-containing amino acid, and the enzyme alliinase [168] and has been reported to prevent arteriosclerosis, stenocardia, cerebral infarction, arrhythmia, and hydrargyria, as well as to enhance the immune system and reduce oxidation $[166,169]$.

Liu et al. found that allicin treatment for glutamateinduced oxidative stress in spinal cord neurons significantly reduced LDH release, loss of cell viability, and apoptotic neuronal death. Allicin effects were associated with reduced oxidative stress, as evidenced by decreased ROS generation, reduced lipid peroxidation, and preservation of antioxidant enzyme activities. Also, allicin diminished the expression of iNOS and significantly increased the expression of heat shock protein 70 (HSP70) at both mRNA and protein levels. Knockdown of HSP70 by specific targeted small interfering RNA (siRNA) not only mitigated allicin-induced protective activity but also partially nullified its effects on the regulation of iNOS [167]. Furthermore, when the beneficial effects of allicin on SCI in mice were investigated, the results showed that allicin significantly increased BBB scores, which was associated with the inhibition of oxidative stress and inflammatory responses. It was also demonstrated that allicin increased the levels of HSP70, increased the phosphorylation of Akt, and reduced the iNOS protein expression levels. Additionally, treatment with allicin significantly reduced ROS and enhanced NADH levels [170]. Liu et al. and Wang and Ren results agreed and collectively demonstrated that the beneficial effects of allicin are mediated by the HSP70/Akt/iNOS pathway and recognized its potential use for SCI treatment $[167,170]$.

4.2.5. Curcumin. Curcumin (1,7-bis(4-hydroxy-3-methoxyphenyl)-1,6-heptadiene-3,5-dione) is a nonsteroidal, naturally occurring compound found in an Indian spice 
commonly used as a dietary pigment known as turmeric. Curcumin exhibits a variety of pharmacologic effects, including anti-inflammatory, anticarcinogenic, anti-infectious, antioxidant, and hypocholesterolemic activities. Diets containing curcumin have shown to stimulate NGF, BDNF, GDNF, and PDGF levels in vivo [171, 172]. Curcumin also enhances neurogenesis and synaptogenesis and improves cognition in rats, as well as in clinical trials for different neurodegenerative diseases [173], probably through promoting these neurotrophic factors.

After spinal cord hemisection, curcumin treatment provides neuroprotection against SCI-induced disability in rats by the attenuation of neuron loss, prevention of neuronal apoptosis, and decreasing astrocyte activation. Curcumin can attenuate astrocyte reactivation in vitro by downregulating GFAP expression, which may improve neuronal survival [174].

\subsection{Extracts from Fruits or Derivatives}

4.3.1. Quercetin. Many fruits and vegetables contain quercetin $\left(3,3^{\prime}, 4^{\prime}, 5,7\right.$-pentahydroxyflavone), a common flavonol [175]. Together with flavones, anthocyanidins, and other compounds, flavonols belong to the class of flavonoids, which in turn represent a major class of polyphenols [176].

Like other polyphenols, quercetin is a scavenger of ROS and reactive nitrogen species such as $\mathrm{NO}$ and ONOO [177]. As well as its metabolites, quercetin acts by modulating the antioxidant defense mechanisms in the cell [178, 179].

The beneficial effects of quercetin on cardiovascular diseases, cancer, infections, inflammatory processes, gastrointestinal tract function, and diabetes have been reported $[177,180,181]$. Moreover, quercetin can exert neuroprotection [182] and antagonize oxidative stress when orally administered in vivo. At a dose of $0.5-50 \mathrm{mg} / \mathrm{kg}$, quercetin protected rodents from oxidative stress and neurotoxicity induced by different insults $[183,184]$. Also, quercetin reduces the immunoreactivity of degenerating neurons [185] and promotes neuronal recovery through the inhibition of inflammatory responses [186].

In a recent study, it was observed that quercetin treatment following acute SCI in rats promoted electrophysiological and locomotor recovery, reduced cavity formation, and contributed to astrocyte activation and axonal regeneration. Additionally, quercetin increased the expression of the brain-derived neurotrophic factor (BDNF), although it reduced $\mathrm{p}$-JNK2 and $\mathrm{p}$-STAT3 expression $[187,188]$. It has been reported that $\mathrm{BDNF}$ activates tropomyosin-related kinase B (Trk-B) through several downstream signaling pathways, such as AKT, CaMK, and Ras/Raf/MEK/ERK, leading to cell survival, growth, and neuroplasticity [189], while the JAK2/STAT3 pathway depends on the binding of erythropoietin (EPO) to a receptor that results in the dimerization of JAK2. This dimerization leads to STAT3 and STAT5 phosphorylation and the formation of stable homodimers and heterodimers, which subsequently induce the transcription of genes that regulate cell proliferation and survival [190]. Consequently, it was proposed that quercetin effects possibly worked through BDNF and JAK2/STAT3 signaling pathways [188].

4.3.2. Tocotrienols. Tocotrienols, isomers of vitamin E, are found in some cereal and vegetable derivatives, such as palm oil, rice bran oil, coconut oil, barley germ, wheat germ, and annatto. Other sources of tocotrienols include grape seed oil, oat, hazelnuts, maize, olive oil, Buckthorn berry, rye, flaxseed oil, poppy seed oil, and sunflower oil [191, 192].

Tocotrienols exhibit strong neuroprotective, antioxidant, and anticancer effects and cholesterol-lowering properties, which are not observed in tocopherols [193]. Due to a better distribution in the lipid layers of the cell membrane, experimental evidence has found that tocotrienols function as better antioxidants and free radical scavengers when compared to tocopherols [194].

In a rat model, tocotrienol protected against SCI by reducing oxidative stress and inflammation and inhibiting iNOS protein expression and activity, as well as plasma NO production. Also, treatment with tocotrienols suppressed TGF- $\beta$, collagen type IV, and fibronectin protein expression levels. Furthermore, the BBB scores in rats treated with $120 \mathrm{mg} / \mathrm{kg} /$ day tocotrienol were significantly higher when compared with the group administered with MPS sodium succinate [38].

4.3.3. Resveratrol. Resveratrol is a naturally occurring stilbene class of polyphenol produced in the skin of many edible plants as a response to fungal infection [195].

The resveratrol-mediated decrease in neuronal MDA levels is often associated with increased activation of antioxidant enzymes such as SOD [196] and antioxidant compounds such as glutathione (GSH) [197].

The antioxidant enzyme HO-1 is implicated particularly as a significant effector of resveratrol-mediated neuroprotection after postischemic reperfusion [198, 199]. Furthermore, resveratrol treatment induces $\mathrm{HO}-1$ expression in cultured mouse cortical neurons [200].

Resveratrol ameliorates kainate-induced excitotoxicity [201]. Subsequently, resveratrol has been shown to improve histopathological and behavioral outcomes after various types of acute CNS injuries, including stroke [202-204], traumatic brain injury $[205,206]$, subarachnoid hemorrhage [207], and SCI [208-210].

In moderate damage to the spinal cord, Liu et al. showed that injured animals treated with resveratrol showed a significant increase in BBB scores. Furthermore, after resveratrol administration, the histopathological analysis showed a restored neuronal morphology and increased the number of neurons. Concerning the antioxidation effects of resveratrol, the treatment overturned the decreased SOD activity and the increased MDA levels caused by SCI, which suggests an antioxidation effect after the injury. Resveratrol treatment also showed an anti-inflammation effect after SCI by inhibiting immunoreactivity and the expression of inflammatory cytokines, such as IL-1 $\beta$, IL-10, TNF- $\alpha$, and myeloperoxidase (MPO). Finally, an antiapoptosis role of resveratrol was observed by the inhibition of injury-induced apoptosis and 
the modulation of the expression of apoptosis-related genes Bax, Bcl-2, and caspase-3 [210].

\subsection{Other Extracts}

4.4.1. Tithonia diversifolia Extracts. As mentioned above, Chinese and European traditional medicine is vast. However, it is not the only option to offer proposals for future acute SCI treatments. An example of African traditional medicine is Tithonia diversifolia, which proved to possess anti-inflammatory properties to treat diabetes mellitus, diarrhea, fever, hematomas, hepatitis, hepatomas, malaria, and wounds $[211,212]$. T. diversifolia is a bushy perennial weed that can be found in Nigerian fields, as well as in wastelands and roadsides in Taiwan.

Phytochemical investigations have identified the existence of some bioactive compounds, such as chromene, flavone, cadinene, germacrene, eudesmane, and rearranged eudesmane derivatives in T. diversifolia [213-215].

Juang et al. obtained $T$. diversifolia ethanolic extracts (TDE) and used it to treat rats with T5 static compression as a model of SCI. First, these researchers noticed that SCI increased the water apparent diffusion coefficient (ADC)-a measurement of the diffusion of water molecules within the central nervous system-after six hours. A low value of $\mathrm{ADC}$ indicates that the nerve fiber tracts are well organized, while a high value means that the tracts are damaged and disorganized. TDE treatment slightly decreased the ADC level after one week in the SCI model. Therefore, it was proposed that TDE protects cells against hydrogen peroxide or radical scavenging-induced toxicity through an antioxidant mechanism, which might be responsible for cell neuroprotection [216].

4.4.2. Danshen Extracts. Danshen (Salvia miltiorrhiza Bunge) is a traditional Chinese herb used for the treatment of heart, liver, and skin diseases, among others. Danshen crude extracts (DCE) attenuate edema and bleeding. Furthermore, DCE treatment improved spinal cord microcirculation, as well as motor function by elevating GDNF mRNA expression in the gray matter of acute SCI in rats [217]. Moreover, DCE increased the expression of the antiapoptotic gene $\mathrm{Bcl}-2$, decreased the number of TUNEL-positive cells, decreased MDA levels, and increased the expression of superoxide dismutase as well [218], which demonstrated that DCE treatment decreased apoptosis and showed beneficial effects over oxidative stress in SCI.

Several chemical components, such as salvianolic acid B (Sal B), 3,4-dihydroxyphenyl lactic acid (DLA), and tanshinone IIA (TIIA), are obtained from Danshen extracts.

Salvianolic acid B (Sal B) is commonly used for the prevention and treatment of cardiovascular disease and shows neuroprotective effects in animal models.

Sal B improves motor function $[219,220]$. One probable mechanism of Sal B is through the oligodendrocyte precursor cell differentiation due to the increase of the myelin sheath and the number of regenerating axons. These observations indicate that Sal B can protect axons and the myelin sheath [221].
Furthermore, Sal B has shown anti-inflammatory effects by attenuating the upregulation of TNF- $\alpha$ and NF- $\kappa \mathrm{B}$ [219]. Moreover, Sal B activates proapoptotic mediators as caspase-3 [220, 221].

Interestingly, Sal B regulates the blood-spinal cord barrier (BSCB) permeability and can reduce spinal edema [220]. In this case, Sal B upregulated the expression of ZO-1 and occludin mediated by $\mathrm{HO}-1$, and p-caveolin was significantly decreased as well $[219,220]$. Additionally, Sal A induced the expression of miR-101, which regulates BSCB integrity via the $\mathrm{miR}-101 / \mathrm{Cul} 3 / \mathrm{Nrf} 2 / \mathrm{HO}-1$ signaling pathway [220].

DLA is obtained by water extraction and improves motor function and tissue damage in SCI. Moreover, it shows its effects on the inflammatory response by reducing $\mathrm{I} \kappa \mathrm{B}-\alpha$ degradation and the nuclear translocation of NF- $\kappa \mathrm{B}$ p 65 subunit, as well as polymorphonuclear cell infiltration and IL-6 production [222].

TIIA is one of the principal components of Danshen, which has shown anti-inflammatory and antiapoptotic effects on several diseases: activates blood circulation and exerts neuroprotective effects. In SCI, TIIA improves motor function and reduces tissue injury [223-225]. A possible mechanism for TIIA is the low activation of astrocytes and upregulated expression of Nestin, NeuN, and NF200, indicating that TIIA can promote cell differentiation [225]. The anti-inflammatory mechanisms are carried out by the inhibition of the activation of NF- $\kappa \mathrm{B}, \mathrm{MAPK}$, and JNK pathways and the downregulation of proinflammatory cytokines TNF- $\alpha$, IL- $1 \beta$, IL- 6 , and iNOS. TIIA decreases neutrophil and monocyte infiltration by decreasing the myeloperoxidase activity [223, 226]. Also, the anti-inflammation induced by TIIA has shown positive responses to neuropathic pain [226].

TIIA reduces apoptosis by decreasing caspase- 3 activation and upregulating Bcl-2 [223, 224, 227]. Finally, TIIA increases the expression of heat-shock protein 70 and inhibits Bax expression [224] and shows effects on redox state imbalance and antiedema [223, 227].

\section{Discussion}

Different cellular and molecular targets are currently under investigation to improve the outcome after SCI. However, no strategies that effectively improve the secondary damage underlying SCI are currently approved by the FDA. Due to the complexity of SCI-in which secondary posttraumatic mechanisms produce neuronal degeneration associated with increased oxidative stress and inflammation-and the lack of therapeutic options, further investigation of other treatments becomes necessary to improve the quality of life of patients with this lesion.

This review describes several compounds derived from plants, vegetables, and fruits that have been tested in SCI models, in which they exhibit antioxidant, anti-inflammatory, and antiapoptotic therapeutic potential. These properties come from compounds such as the asiatic acid, obtained from Centella asiatica, and plumbagin, an analog of vitamin K3 isolated from Plumbago zeylanica L, which increase the levels of antioxidant enzymes. Ligustilide is a 
bioactive ingredient that reduces oxidative stress and inflammation. Tetrandrine, which is extracted from the root of Stephania tetrandrae, is a compound with neuroprotective effects through blocking calcium canals. Consequently, it reduces the molecules associated with oxidative stress damage. Danshen extracts decreased apoptosis and oxidative stress and improved motor function in acute SCI. Ginsenosides extracted from $P$. ginseng promote neuronal restoration, inhibit inflammatory processes, and downregulate oxidative stress.

Puerarin, the main constituent of Radix Puerariae lobata, and tetramethylpyrazine, extracted from Ligusticum wallichii Franchat, showed neuroprotective, as well as antioxidant and antiapoptosis effects, and also reduced neuroinflammation against SCI.

EGb-761, an extract from Ginkgo biloba, improved functional performance after SCI, due to its antioxidant, free radical scavenging, and antiapoptosis properties.

Some polyphenols, including quercetin, rosmarinic acid, silymarin, epigallocatechin-3-gallate, and resveratrol, are ROS and reactive nitrogen species scavengers. Therefore, they contribute to regulating oxidative stress. Lastly, curcumin is a nonsteroidal compound with a variety of pharmacological effects.

Some limitations of this review were the lack of information about the use of other natural compounds from Mexican and Latin American countries-which are known to possess an impactful millennial tradition in the use of medicinal plants-to treat SCI. However, this opens up the possibility of future research for new American natural compounds with antioxidant properties, which could be used as a potential treatment for SCI as well. Due to the heterogeneity between the beginning, the duration, and the routes of administration of the treatments, it is impossible to compare their efficiency and strength. Therefore, another limitation could be the lack of standard procedures that allow the comparison of the effectiveness of these compounds.

Although many natural compounds have been used in SCI, little is known about their effectiveness as natural antioxidants, the mechanisms through which these compounds exert their antioxidant activities or their ability to cross the $\mathrm{BBB}$ in preclinical models.

As a conclusion, 21 compounds commonly used in SCI models with beneficial properties were described in this review. These compounds are potential therapeutic candidates with neuroprotective effects such as reducing the levels of ROS and diminishing oxidative stress.

Even though these compounds have been tested in animal models with promising results, no clinical studies have been conducted in humans. Therefore, it is crucial to design some strategies to study the effects of these natural compounds in patients with SCI, given that most of these plants are available worldwide at a much lower cost than some synthetic drugs used for SCI therapy.

\section{Conflicts of Interest}

The authors declare no conflicts of interest regarding the publication of this paper.

\section{Acknowledgments}

The authors wish to thank the Programa Cátedras CONACYT and the Hospital de Especialidades Bernardo Sepúlveda, Centro Médico Nacional SXXI, Instituto Mexicano del Seguro Social. The authors financed this review with resources of their own.

\section{References}

[1] National SCI Statistical Centerhttps://www.nscisc.uab.edu.

[2] M. J. DeVivo, "Epidemiology of traumatic spinal cord injury: trends and future implications," Spinal Cord, vol. 50, no. 5, pp. 365-372, 2012.

[3] P. E. Ludwig, A. A. Patil, A. J. Chamczuk, and D. K. Agrawal, "Hormonal therapy in traumatic spinal cord injury," American Journal of Translational Research, vol. 9, no. 9, pp. 3881-3895, 2017.

[4] S. Samantaray, A. Das, D. C. Matzelle et al., "Administration of low dose estrogen attenuates gliosis and protects neurons in acute spinal cord injury in rats," Journal of Neurochemistry, vol. 136, no. 5, pp. 1064-1073, 2016.

[5] H. Q. Cao and E. D. Dong, "An update on spinal cord injury research," Neuroscience Bulletin, vol. 29, no. 1, pp. 94-102, 2013.

[6] W. H. Donovan, "Spinal cord injury-past, present, and future," The Journal of Spinal Cord Medicine, vol. 30, no. 2, pp. 85-100, 2007.

[7] E. Pickelsimer, E. J. Shiroma, and D. A. Wilson, "Statewide investigation of medically attended adverse health conditions of persons with spinal cord injury," The Journal of Spinal Cord Medicine, vol. 33, no. 3, pp. 221-231, 2010.

[8] M. G. Fehlings and C. H. Tator, "The relationships among the severity of spinal cord injury, residual neurological function, axon counts, and counts of retrogradely labeled neurons after experimental spinal cord injury," Experimental Neurology, vol. 132, no. 2, pp. 220-228, 1995.

[9] J. W. McDonald and C. Sadowsky, "Spinal-cord injury," The Lancet, vol. 359, no. 9304, pp. 417-425, 2002.

[10] A. Ackery, C. Tator, and A. Krassioukov, "A global perspective on spinal cord injury epidemiology," Journal of Neurotrauma, vol. 21, no. 10, pp. 1355-1370, 2004.

[11] P. K. Yip and A. Malaspina, "Spinal cord trauma and the molecular point of no return," Molecular Neurodegeneration, vol. 7, no. 1, p. 6, 2012.

[12] M. D. Norenberg, J. Smith, and A. Marcillo, "The pathology of human spinal cord injury: defining the problems," Journal of Neurotrauma, vol. 21, no. 4, pp. 429-440, 2004.

[13] S. C. Cramer, L. Lastra, M. G. Lacourse, and M. J. Cohen, "Brain motor system function after chronic, complete spinal cord injury," Brain, vol. 128, Part 12, pp. 2941-2950, 2005.

[14] G. Yiu and Z. He, "Glial inhibition of CNS axon regeneration," Nature Reviews Neuroscience, vol. 7, no. 8, pp. 617627, 2006.

[15] A. Ulndreaj, J. C. Chio, C. S. Ahuja, and M. G. Fehlings, "Modulating the immune response in spinal cord injury," Expert Review of Neurotherapeutics, vol. 16, no. 10, pp. 1127-1129, 2016.

[16] S. J. Cooney, Y. Zhao, and K. R. Byrnes, "Characterization of the expression and inflammatory activity of NADPH oxidase 
after spinal cord injury," Free Radical Research, vol. 48, no. 8, pp. 929-939, 2014.

[17] I. Jamme, E. Petit, D. Divoux, A. Gerbi, J. M. Maixent, and A. Nouvelot, "Modulation of mouse cerebral Na+, K(+)ATPase activity by oxygen free radicals," NeuroReport, vol. 7, no. 1, pp. 333-337, 1995.

[18] M. Liu, W. Wu, H. Li et al., "Necroptosis, a novel type of programmed cell death, contributes to early neural cells damage after spinal cord injury in adult mice," The Journal of Spinal Cord Medicine, vol. 38, no. 6, pp. 745-753, 2015.

[19] Y. Wang, H. Wang, Y. Tao, S. Zhang, J. Wang, and X. Feng, "Necroptosis inhibitor necrostatin-1 promotes cell protection and physiological function in traumatic spinal cord injury," Neuroscience, vol. 266, pp. 91-101, 2014.

[20] S. Li and P. K. Stys, "Mechanisms of ionotropic glutamate receptor-mediated excitotoxicity in isolated spinal cord white matter," The Journal of Neuroscience, vol. 20, no. 3, pp. 11901198, 2000.

[21] V. Gallo, A. Bertolotto, and G. Levi, "The proteoglycan chondroitin sulfate is present in a subpopulation of cultured astrocytes and in their precursors," Developmental Biology, vol. 123, no. 1, pp. 282-285, 1987.

[22] L. L. Jones, R. U. Margolis, and M. H. Tuszynski, “The chondroitin sulfate proteoglycans neurocan, brevican, phosphacan, and versican are differentially regulated following spinal cord injury," Experimental Neurology, vol. 182, no. 2, pp. 399-411, 2003.

[23] R. Katoh-Semba, M. Matsuda, K. Kato, and A. Oohira, "Chondroitin Sulphate Proteoglycans in the Rat Brain: Candidates for Axon Barriers of Sensory Neurons and the Possible Modification by Laminin of their Actions," The European Journal of Neuroscience, vol. 7, no. 4, pp. 613-621, 1995.

[24] M. B. Bracken and T. R. Holford, "Neurological and functional status 1 year after acute spinal cord injury: estimates of functional recovery in National Acute Spinal Cord Injury Study II from results modeled in National Acute Spinal Cord Injury Study III," Journal of Neurosurgery, vol. 96, 3 Supplement, pp. 259-266, 2002.

[25] N. A. Silva, N. Sousa, R. L. Reis, and A. J. Salgado, "From basics to clinical: a comprehensive review on spinal cord injury," Progress in Neurobiology, vol. 114, pp. 25-57, 2014.

[26] D. R. Sengelaub and X. M. Xu, "Protective effects of gonadal hormones on spinal motoneurons following spinal cord injury," Neural Regeneration Research, vol. 13, no. 6, pp. 971-976, 2018.

[27] O. H. Bedreag, A. F. Rogobete, M. Sărăndan et al., "Oxidative stress and antioxidant therapy in traumatic spinal cord injuries," Romanian Journal of Anaesthesia and Intensive Care, vol. 21, no. 2, pp. 123-129, 2014.

[28] M. Bains and E. D. Hall, "Antioxidant therapies in traumatic brain and spinal cord injury," Biochimica et Biophysica Acta, vol. 1822, no. 5, pp. 675-684, 2012.

[29] E. D. Hall, "Antioxidant therapies for acute spinal cord injury," Neurotherapeutics, vol. 8, no. 2, pp. 152-167, 2011.

[30] E. D. Hall, J. A. Wang, D. M. Miller, J. E. Cebak, and R. L. Hill, "Newer pharmacological approaches for antioxidant neuroprotection in traumatic brain injury," Neuropharmacology, vol. 145, Part B, pp. 247-258, 2019.

[31] J. M. Gutteridge, "Lipid peroxidation and antioxidants as biomarkers of tissue damage," Clinical Chemistry, vol. 41, no. 12, Part 2, pp. 1819-1828, 1995.
[32] M. L. Seligman, E. S. Flamm, B. D. Goldstein, R. G. Poser, H. B. Demopoulos, and J. Ransohoff, "Spectrofluorescent detection of malonaldehyde as a measure of lipid free radical damage in response to ethanol potentiation of spinal cord trauma," Lipids, vol. 12, no. 11, pp. 945-950, 1977.

[33] S. A. Baldwin, R. Broderick, D. Osbourne, G. Waeg, D. A. Blades, and S. W. Scheff, "The presence of 4-hydroxynonenal/protein complex as an indicator of oxidative stress after experimental spinal cord contusion in a rat model," Journal of Neurosurgery, vol. 88, no. 5, pp. 874-883, 1998.

[34] J. Luo, K. Uchida, and R. Shi, "Accumulation of acroleinprotein adducts after traumatic spinal cord injury," Neurochemical Research, vol. 30, no. 3, pp. 291-295, 2005.

[35] A. T. Michael-Titus, "Omega-3 fatty acids and neurological injury," Prostaglandins, Leukotrienes, and Essential Fatty Acids, vol. 77, no. 5-6, pp. 295-300, 2007.

[36] H. Ahsan, "3-Nitrotyrosine: A biomarker of nitrogen free radical species modified proteins in systemic autoimmunogenic conditions," Human Immunology, vol. 74, no. 10, pp. 1392-1399, 2013.

[37] Y. Xiong, A. G. Rabchevsky, and E. D. Hall, "Role of peroxynitrite in secondary oxidative damage after spinal cord injury," Journal of Neurochemistry, vol. 100, no. 3, pp. 639649, 2007.

[38] C. Xun, M. Mamat, H. Guo et al., "Tocotrienol alleviates inflammation and oxidative stress in a rat model of spinal cord injury via suppression of transforming growth factor$\beta$," Experimental and Therapeutic Medicine, vol. 14, no. 1, pp. 431-438, 2017.

[39] Q. Zhang, H. Yang, J. An, R. Zhang, B. Chen, and D. J. Hao, "Therapeutic Effects of Traditional Chinese Medicine on Spinal Cord Injury: A Promising Supplementary Treatment in Future," Evidence-based Complementary and Alternative Medicine, vol. 2016, Article ID 8958721, 18 pages, 2016.

[40] M. F. Nagoor Meeran, S. N. Goyal, K. Suchal, C. Sharma, C. R. Patil, and S. K. Ojha, "Pharmacological properties, molecular mechanisms, and pharmaceutical development of asiatic acid: a pentacyclic triterpenoid of therapeutic promise," Frontiers in Pharmacology, vol. 9, p. 892, 2018.

[41] W. Jiang, M. Li, F. He et al., "Neuroprotective effect of asiatic acid against spinal cord injury in rats," Life Sciences, vol. 157, pp. 45-51, 2016.

[42] A. Loboda, M. Damulewicz, E. Pyza, A. Jozkowicz, and J. Dulak, "Role of Nrf2/HO-1 system in development, oxidative stress response and diseases: an evolutionarily conserved mechanism," Cellular and Molecular Life Sciences, vol. 73, no. 17, pp. 3221-3247, 2016.

[43] J. Tschopp and K. Schroder, "NLRP3 inflammasome activation: the convergence of multiple signalling pathways on ROS production?," Nature Reviews Immunology, vol. 10, no. 3, pp. 210-215, 2010.

[44] W. Jiang, M. Li, F. He et al., "Protective effects of asiatic acid against spinal cord injury-induced acute lung injury in rats," Inflammation, vol. 39, no. 6, pp. 1853-1861, 2016.

[45] Y. Chen, Y. Yu, C. Chen, W. Yang, C. Wang, and J. R. Du, "Pharmacokinetic profile of Z-ligustilide in rat plasma and brain following oral administration," Natural Product Research and Development, vol. 22, pp. 126-131, 2010.

[46] X. Kuang, Y. Yao, J. R. Du, Y. X. Liu, C. Y. Wang, and Z. M. Qian, "Neuroprotective role of Z-ligustilide against forebrain 
ischemic injury in ICR mice," Brain Research, vol. 1102, no. 1, pp. 145-153, 2006.

[47] X. Kuang, J. Du, Y. Liu, G. Zhang, and H. Peng, "Postischemic administration of Z-Ligustilide ameliorates cognitive dysfunction and brain damage induced by permanent forebrain ischemia in rats," Pharmacology, Biochemistry, and Behavior, vol. 88, no. 3, pp. 213-221, 2008.

[48] X. Kuang, L. F. Wang, L. Yu et al., "Ligustilide ameliorates neuroinflammation and brain injury in focal cerebral ische$\mathrm{mia} /$ reperfusion rats: involvement of inhibition of TLR4/peroxiredoxin 6 signaling," Free Radical Biology \& Medicine, vol. 71, pp. 165-175, 2014.

[49] P. O. Donkor, Y. Chen, L. Ding, and F. Qiu, "Locally and traditionally used Ligusticum species - A review of their phytochemistry, pharmacology and pharmacokinetics," Journal of Ethnopharmacology, vol. 194, pp. 530-548, 2016.

[50] W. Xiao, A. Yu, D. Liu, J. Shen, and Z. Xu, "Ligustilide treatment promotes functional recovery in a rat model of spinal cord injury via preventing ROS production," International Journal of Clinical and Experimental Pathology, vol. 8, no. 10, pp. 12005-12013, 2015.

[51] L. Fan, K. Wang, Z. Shi, J. Die, C. Wang, and X. Dang, "Tetramethylpyrazine protects spinal cord and reduces inflammation in a rat model of spinal cord ischemia-reperfusion injury," Journal of Vascular Surgery, vol. 54, no. 1, pp. 192200, 2011.

[52] J. Z. Hu, J. H. Huang, Z. M. Xiao, J. H. Li, X. M. Li, and H. B. $\mathrm{Lu}$, "Tetramethylpyrazine accelerates the function recovery of traumatic spinal cord in rat model by attenuating inflammation," Journal of the Neurological Sciences, vol. 324, no. 1-2, pp. 94-99, 2013.

[53] S. Wang, A. Li, and S. Guo, "Ligustrazine attenuates neuropathic pain by inhibition of JAK/STAT3 pathway in a rat model of chronic constriction injury," Pharmazie, vol. 71, no. 7, pp. 408-412, 2016.

[54] J. W. Shin, J. Y. Moon, J. W. Seong et al., "Effects of tetramethylpyrazine on microglia activation in spinal cord compression injury of mice," The American Journal of Chinese Medicine, vol. 41, no. 6, pp. 1361-1376, 2013.

[55] C. Wang, P. Wang, W. Zeng, and W. Li, "Tetramethylpyrazine improves the recovery of spinal cord injury via Akt/Nrf2/HO-1 pathway," Bioorganic \& Medicinal Chemistry Letters, vol. 26, no. 4, pp. 1287-1291, 2016.

[56] J. Yang, S. Yang, and Y. J. Yuan, "Integrated investigation of lipidome and related signaling pathways uncovers molecular mechanisms of tetramethylpyrazine and butylidenephthalide protecting endothelial cells under oxidative stress," Molecular BioSystems, vol. 8, no. 6, pp. 1789-1797, 2012.

[57] J. Hu, Y. Lang, Y. Cao, T. Zhang, and H. Lu, "The neuroprotective effect of tetramethylpyrazine against contusive spinal cord injury by activating PGC- $1 \alpha$ in rats," Neurochemical Research, vol. 40, no. 7, pp. 1393-1401, 2015.

[58] J. Hu, Y. Cao, T. Wu, D. Li, and H. Lu, "Micro-CT as a tool to investigate the efficacy of tetramethylpyrazine in a rat spinal cord injury model," Spine, vol. 41, no. 16, pp. 1272-1278, 2016.

[59] J. H. Huang, Y. Cao, L. Zeng et al., “Tetramethylpyrazine enhances functional recovery after contusion spinal cord injury by modulation of microRNA-21, FasL, PDCD4 and PTEN expression," Brain Research, vol. 1648, Part A, pp. 35-45, 2016.
[60] J. Z. Hu, X. K. Wang, Y. Cao et al., "Tetramethylpyrazine facilitates functional recovery after spinal cord injury by inhibiting MMP2, MMP9, and vascular endothelial cell apoptosis," Current Neurovascular Research, vol. 14, no. 2, pp. 110-116, 2017.

[61] Y. F. Leng, X. M. Gao, S. X. Wang, and Y. H. Xing, "Effects of tetramethylpyrazine on neuronal apoptosis in the superficial dorsal horn in a rat model of neuropathic pain," The American Journal of Chinese Medicine, vol. 40, no. 6, pp. 12291239, 2012.

[62] L. Jiang, C. L. Pan, C. Y. Wang et al., "Selective suppression of the JNK-MMP2/9 signal pathway by tetramethylpyrazine attenuates neuropathic pain in rats," Journal of Neuroinflammation, vol. 14, no. 1, p. 174, 2017.

[63] Y. Fan and Y. Wu, "Tetramethylpyrazine alleviates neural apoptosis in injured spinal cord via the downregulation of miR-214-3p," Biomedicine \& Pharmacotherapy, vol. 94, pp. 827-833, 2017.

[64] A. R. Khalatbary, T. Tiraihi, M. B. Boroujeni, H. Ahmadvand, M. Tavafi, and A. Tamjidipoor, "Effects of epigallocatechin gallate on tissue protection and functional recovery after contusive spinal cord injury in rats," Brain Research, vol. 1306, pp. 168-175, 2010.

[65] A. R. Khalatbary and H. Ahmadvand, "Anti-inflammatory effect of the epigallocatechin gallate following spinal cord trauma in rat," Iranian Biomedical Journal, vol. 15, no. 1-2, pp. 31-37, 2011.

[66] W. Tian, X. G. Han, Y. J. Liu et al., "Intrathecal epigallocatechin gallate treatment improves functional recovery after spinal cord injury by upregulating the expression of BDNF and GDNF," Neurochemical Research, vol. 38, no. 4, pp. 772-779, 2013.

[67] W. M. Renno, G. Al-Khaledi, A. Mousa, S. M. Karam, H. Abul, and S. Asfar, "(-)-Epigallocatechin-3-gallate (EGCG) modulates neurological function when intravenously infused in acute and, chronically injured spinal cord of adult rats," Neuropharmacology, vol. 77, pp. 100-119, 2014.

[68] W. M. Renno, M. Al-Maghrebi, M. S. Rao, and H. Khraishah, “(-)-Epigallocatechin-3-gallate modulates spinal cord neuronal degeneration by enhancing growth-associated protein 43, B-cell lymphoma 2, and decreasing B-cell lymphoma 2associated $\mathrm{x}$ protein expression after sciatic nerve crush injury," Journal of Neurotrauma, vol. 32, no. 3, pp. 170$184,2015$.

[69] W. M. Renno, K. M. Khan, and L. Benov, "Is there a role for neurotrophic factors and their receptors in augmenting the neuroprotective effect of (-)-epigallocatechin-3-gallate treatment of sciatic nerve crush injury?," Neuropharmacology, vol. 102, pp. 1-20, 2016.

[70] L. Machova Urdzikova, J. Ruzicka, K. Karova et al., “A green tea polyphenol epigallocatechin-3-gallate enhances neuroregeneration after spinal cord injury by altering levels of inflammatory cytokines," Neuropharmacology, vol. 126, pp. 213-223, 2017.

[71] U. Gundimeda, T. H. McNeill, B. A. Barseghian et al., "Polyphenols from green tea prevent antineuritogenic action of Nogo-A via $67-\mathrm{kDa}$ laminin receptor and hydrogen peroxide," Journal of Neurochemistry, vol. 132, no. 1, pp. 70-84, 2015.

[72] R. J. Pasterkamp and J. Verhaagen, "Semaphorins in axon regeneration: developmental guidance molecules gone 
wrong?," Philosophical Transactions of the Royal Society of London. Series B, Biological Sciences, vol. 361, no. 1473, pp. 1499-1511, 2006.

[73] X. Kuang, Y. Huang, H. F. Gu et al., "Effects of intrathecal epigallocatechin gallate, an inhibitor of Toll-like receptor 4, on chronic neuropathic pain in rats," European Journal of Pharmacology, vol. 676, no. 1-3, pp. 51-56, 2012.

[74] B. Álvarez-Pérez, J. Homs, M. Bosch-Mola et al., "Epigallocatechin-3-gallate treatment reduces thermal hyperalgesia after spinal cord injury by down-regulating RhoA expression in mice," European Journal of Pain, vol. 20, no. 3, pp. 341352, 2016.

[75] M. Bosch-Mola, J. Homs, B. Álvarez-Pérez et al., “(-)-Epigallocatechin-3-Gallate Antihyperalgesic Effect Associates With Reduced CX3CL1 Chemokine Expression in Spinal Cord," Phytotherapy Research, vol. 31, no. 2, pp. 340-344, 2017.

[76] X. Xifró, L. Vidal-Sancho, P. Boadas-Vaello et al., "Novel epigallocatechin-3-gallate (EGCG) derivative as a new therapeutic strategy for reducing neuropathic pain after chronic constriction nerve injury in mice," PLoS One, vol. 10, no. 4, article e0123122, 2015.

[77] W. M. Renno, L. Benov, and K. M. Khan, "Possible role of antioxidative capacity of (-)-epigallocatechin-3-gallate treatment in morphological and neurobehavioral recovery after sciatic nerve crush injury," Journal of Neurosurgery. Spine, vol. 27, no. 5, pp. 593-613, 2017.

[78] T. Kaku, T. Miyata, T. Uruno, I. Sako, and Y. Kinoshita, "Chemico-pharmacological studies on saponins of Panax ginseng C. A. Meyer," Arzneimittel-Forschung, vol. 25, pp. 539-547, 1975.

[79] T. H. Lan, Z. W. Xu, Z. Wang, Y. L. Wu, W. K. Wu, and H. M. Tan, "Ginsenoside Rb1 prevents homocysteine-induced endothelial dysfunction via PI3K/Akt activation and PKC inhibition," Biochemical Pharmacology, vol. 82, no. 2, pp. 148-155, 2011.

[80] T. Ahmed, S. H. Raza, A. Maryam et al., "Ginsenoside Rb1 as a neuroprotective agent: a review," Brain Research Bulletin, vol. 125 , pp. $30-43,2016$.

[81] B. Liao, H. Newmark, and R. Zhou, "Neuroprotective Effects of Ginseng Total Saponin and Ginsenosides Rb1 and Rg1 on Spinal Cord Neurons in Vitro," Experimental Neurology, vol. 173, no. 2, pp. 224-234, 2002.

[82] G. X. Chu and X. Chen, "Anti-lipid peroxidation and protection of ginsenosides against cerebral ischemia-reperfusion injuries in rats," Zhongguo Yao Li Xue Bao, vol. 11, no. 2, pp. 119-123, 1990.

[83] Y. C. Kim, S. R. Kim, G. J. Markelonis, and T. H. Oh, "Ginsenosides $\mathrm{Rb} 1$ and $\mathrm{Rg} 3$ protect cultured rat cortical cells from glutamate-induced neurodegeneration," Journal of Neuroscience Research, vol. 53, no. 4, pp. 426-432, 1998.

[84] J. Ye, J. P. Yao, X. Wang et al., "Neuroprotective effects of ginsenosides on neural progenitor cells against oxidative injury," Molecular Medicine Reports, vol. 13, no. 4, pp. 3083-3091, 2016.

[85] N. Ni, Q. Liu, H. Ren et al., "Ginsenoside Rb1 protects rat neural progenitor cells against oxidative injury," Molecules, vol. 19, no. 3, pp. 3012-3024, 2014.

[86] B. Zhang, R. Hata, P. Zhu et al., "Prevention of ischemic neuronal death by intravenous infusion of a ginseng saponin, ginsenoside $\mathrm{Rb}(1)$, that upregulates $\mathrm{Bcl}-\mathrm{x}(\mathrm{L})$ expression,"
Journal of Cerebral Blood Flow and Metabolism, vol. 26, no. 5, pp. 708-721, 2006.

[87] R. Collins, "A ten-year audit of traditional Chinese medicine and other natural product research published in the Chinese Medical Journal (2000-2009)," Chinese Medical Journal, vol. 124, no. 9, pp. 1401-1408, 2011.

[88] Y. J. Kim, D. Zhang, and D. C. Yang, "Biosynthesis and biotechnological production of ginsenosides," Biotechnology Advances, vol. 33, no. 6, Part 1, pp. 717-735, 2015.

[89] C. H. Lee and J. H. Kim, "A review on the medicinal potentials of ginseng and ginsenosides on cardiovascular diseases," Journal of Ginseng Research, vol. 38, no. 3, pp. 161-166, 2014.

[90] M. Sakanaka, P. Zhu, B. Zhang et al., "Intravenous infusion of dihydroginsenoside Rb1 prevents compressive spinal cord injury and ischemic brain damage through upregulation of VEGF and Bcl-XL," Journal of Neurotrauma, vol. 24, no. 6, pp. 1037-1054, 2007.

[91] Y. O. Kim, Y. Kim, K. Lee et al., "Panax ginseng Improves Functional Recovery after Contusive Spinal Cord Injury by Regulating the Inflammatory Response in Rats: An In Vivo Study," Evidence-based Complementary and Alternative Medicine, vol. 2015, Article ID 817096, 7 pages, 2015.

[92] P. Zhu, K. Samukawa, H. Fujita, H. Kato, and M. Sakanaka, "Oral administration of red ginseng extract promotes neurorestoration after compressive spinal cord injury in rats," Evidence-based Complementary and Alternative Medicine, vol. 2017, Article ID 1265464, 10 pages, 2017.

[93] W. Wang, H. Shen, J. J. Xie, J. Ling, and H. Lu, "Neuroprotective effect of ginseng against spinal cord injury-induced oxidative stress and inflammatory responses," International Journal of Clinical and Experimental Medicine, vol. 8, no. 3, pp. 3514-3521, 2015.

[94] R. Uzayisenga, P. A. Ayeka, and Y. Wang, "Anti-Diabetic Potential of Panax Notoginseng Saponins (PNS): A Review," Phytotherapy Research, vol. 28, no. 4, pp. 510-516, 2014.

[95] X. Yang, X. Xiong, H. Wang, and J. Wang, "Protective effects of Panax notoginseng saponins on cardiovascular diseases: a comprehensive overview of experimental studies," Evidencebased Complementary and Alternative Medicine, vol. 2014, Article ID 204840, 13 pages, 2014.

[96] N. Zhou, Y. Tang, R. F. Keep, X. Ma, and J. Xiang, "Antioxidative effects of Panax notoginseng saponins in brain cells," Phytomedicine, vol. 21, no. 10, pp. 1189-1195, 2014.

[97] T. Wang, R. Guo, G. Zhou et al., "Traditional uses, botany, phytochemistry, pharmacology and toxicology of Panax notoginseng (Burk.) F.H. Chen: A review," Journal of Ethnopharmacology, vol. 188, pp. 234-258, 2016.

[98] Z. H. Chen, J. Li, J. Liu et al., "Saponins isolated from the root of Panax notoginseng showed significant anti-diabetic effects in KK-Ay mice," The American Journal of Chinese Medicine, vol. 36, no. 5, pp. 939-951, 2008.

[99] M. Zheng, L. Qu, and Y. Lou, "Effects of icariin combined with Panax notoginseng saponins on ischemia reperfusioninduced cognitive impairments related with oxidative stress and CA1 of hippocampal neurons in rat," Phytotherapy Research, vol. 22, no. 5, pp. 597-604, 2008.

[100] H. Li, C. Q. Deng, B. Y. Chen, S. P. Zhang, Y. Liang, and X. G. Luo, "Total saponins of Panax Notoginseng modulate the expression of caspases and attenuate apoptosis in rats following focal cerebral ischemia-reperfusion," Journal of Ethnopharmacology, vol. 121, no. 3, pp. 412-418, 2009. 
[101] N. Ning, X. Dang, C. Bai, C. Zhang, and K. Wang, "Panax notoginsenoside produces neuroprotective effects in rat model of acute spinal cord ischemia-reperfusion injury," Journal of Ethnopharmacology, vol. 139, no. 2, pp. 504-512, 2012.

[102] B. P. Jacobs and W. S. Browner, "Ginkgo biloba: a living fossil," The American Journal of Medicine, vol. 108, no. 4, pp. 341-342, 2000.

[103] J. V. Smith and Y. Luo, "Studies on molecular mechanisms of Ginkgo biloba extract," Applied Microbiology and Biotechnology, vol. 64, no. 4, pp. 465-472, 2004.

[104] A. J. Kellermann and C. Kloft, "Is There a Risk of Bleeding Associated with Standardized Ginkgo bilobaExtract Therapy? A Systematic Review and Meta-analysis," Pharmacotherapy, vol. 31, no. 5, pp. 490-502, 2011.

[105] T. Yoshitake, S. Yoshitake, and J. Kehr, "The Ginkgo biloba extract EGb $761^{\circledR}$ and its main constituent flavonoids and ginkgolides increase extracellular dopamine levels in the rat prefrontal cortex," British Journal of Pharmacology, vol. 159, no. 3, pp. 659-668, 2010.

[106] F. Tchantchou, P. N. Lacor, Z. Cao et al., "Stimulation of neurogenesis and synaptogenesis by bilobalide and quercetin via common final pathway in hippocampal neurons," Journal of Alzheimer's Disease, vol. 18, no. 4, pp. 787798, 2009.

[107] P. Montes, E. Ruiz-Sanchez, C. Rojas, and P. Rojas, "Ginkgo biloba extract 761: a review of basic studies and potential clinical use in psychiatric disorders," CNS \& Neurological Disorders Drug Targets, vol. 14, no. 1, pp. 132-149, 2015.

[108] C. Shi, S. Xiao, J. Liu et al., "Ginkgo biloba extract EGb761 protects against aging-associated mitochondrial dysfunction in platelets and hippocampi of SAMP8 mice," Platelets, vol. 21, no. 5, pp. 373-379, 2010.

[109] J. H. Cho, J. H. Sung, E. H. Cho et al., "Gingko biloba extract (EGb 761) prevents ischemic brain injury by activation of the Akt signaling pathway," The American Journal of Chinese Medicine, vol. 37, no. 3, pp. 547-555, 2009.

[110] F. DeFeudis and K. Drieu, "Ginkgo biloba extract (EGb 761) and CNS functions: basic studies and clinical applications," Current Drug Targets, vol. 1, no. 1, pp. 25-58, 2000.

[111] S. E. Nada, J. Tulsulkar, and Z. A. Shah, "Heme oxygenase 1mediated neurogenesis is enhanced by Ginkgo biloba (EGb $761^{\circledR}$ ) after permanent ischemic stroke in mice," Molecular Neurobiology, vol. 49, no. 2, pp. 945-956, 2014.

[112] L. Brunetti, B. Michelotto, G. Orlando et al., "Aging increases amyloid $\beta$-peptide-induced 8-iso-prostaglandin $\mathrm{F}_{2 \alpha}$ release from rat brain," Neurobiology of Aging, vol. 25, no. 1, pp. 125-129, 2004.

[113] N. E. Mohamed and A. E. Abd El-Moneim, "Ginkgo biloba extract alleviates oxidative stress and some neurotransmitters changes induced by aluminum chloride in rats," Nutrition, vol. 35, pp. 93-99, 2017.

[114] Y. Oyama, P. A. Fuchs, N. Katayama, and K. Noda, "Myricetin and quercetin, the flavonoid constituents of Ginkgo biloba extract, greatly reduce oxidative metabolism in both resting and $\mathrm{Ca}^{2+}$-loaded brain neurons," Brain Research, vol. 635, no. 1-2, pp. 125-129, 1994.

[115] Y. Oyama, L. Chikahisa, T. Ueha, K. Kanemaru, and K. Noda, "Ginkgo biloba extract protects brain neurons against oxidative stress induced by hydrogen peroxide," Brain Research, vol. 712, no. 2, pp. 349-352, 1996.
[116] L. Marcocci, L. Packer, M. T. Droy-Lefaix, A. Sekaki, and M. Gardès-Albert, "Antioxidant action of Ginkgo biloba extract EGb-761," Methods in Enzymology, vol. 46, pp. 462475, 1994.

[117] W. Gsell, N. Reichert, M. B. Youdim, and P. Riederer, "Interaction of neuroprotective substances with human brain superoxide dismutase. An in vitro study," Journal of Neural Transmission, vol. 45, pp. 271-279, 1995.

[118] J. Pincemail, M. Dupuis, C. Nasr et al., "Superoxide anion scavenging effect and superoxide dismutase activity of Ginkgo biloba extract," Experientia, vol. 45, no. 8, pp. 708712, 1989.

[119] I. Maitra, L. Marcocci, M. T. Droy-Lefaix, and L. Packer, "Peroxyl radical scavenging activity of Ginkgo biloba extract EGb 761,” Biochemical Pharmacology, vol. 49, no. 11, pp. 1649-1655, 1995.

[120] E. Mechirova, I. Domorakova, M. Dankova, V. Danielisova, and J. Burda, "Effect of Noradrenalin and EGb 761 pretreatment on the ischemia-reperfusion injured spinal cord neurons in rabbits," Cellular and Molecular Neurobiology, vol. 29, no. 6-7, pp. 991-998, 2009.

[121] M. Yan, Y. W. Liu, W. Shao et al., "EGb761 improves histological and functional recovery in rats with acute spinal cord contusion injury," Spinal Cord, vol. 54, no. 4, pp. 259-265, 2016.

[122] V. S. Kotakadi, Y. Jin, A. B. Hofseth et al., "Ginkgo biloba extract EGb 761 has anti-inflammatory properties and ameliorates colitis in mice by driving effector T cell apoptosis," Carcinogenesis, vol. 29, no. 9, pp. 1799-1806, 2008.

[123] X. Jiang, B. Nie, S. Fu et al., "EGb761 protects hydrogen peroxide-induced death of spinal cord neurons through inhibition of intracellular ROS production and modulation of apoptotic regulating genes," Journal of Molecular Neuroscience, vol. 38, no. 2, pp. 103-113, 2009.

[124] J. Wang, C. Ma, W. Rong et al., "Bog bilberry anthocyanin extract improves motor functional recovery by multifaceted effects in spinal cord injury," Neurochemical Research, vol. 37, no. 12, pp. 2814-2825, 2012.

[125] R. K. Koc, H. Akdemir, A. Kurtsoy et al., "Lipid peroxidation in experimental spinal cord injury. Comparison of treatment with Ginkgo biloba, TRH and methylprednisolone," Research in Experimental Medicine, vol. 195, no. 2, pp. 117-123, 1995.

[126] L. H. Fan, K. Z. Wang, and B. Cheng, "Effects of Ginkgo biloba extract on lipid peroxidation and apoptosis after spinal cord ischemia/reperfusion in rabbits," Chinese Journal of Traumatology, vol. 9, no. 2, pp. 77-81, 2006.

[127] Z. Zhao, N. Liu, J. Huang, P. H. Lu, and X. M. Xu, "Inhibition of cPLA $_{2}$ activation by Ginkgo biloba extract protects spinal cord neurons from glutamate excitotoxicity and oxidative stress-induced cell death," Journal of Neurochemistry, vol. 116, no. 6, pp. 1057-1065, 2011.

[128] S. Badem, M. Ugurlucan, H. El et al., "Effects of Ginkgo biloba Extract on Spinal Cord Ischemia -Reperfusion Injury in Rats," Annals of Vascular Surgery, vol. 28, no. 5, pp. 12961305, 2014.

[129] Q. Ao, X. H. Sun, A. J. Wang et al., "Protective effects of extract of Ginkgo biloba (EGb 761) on nerve cells after spinal cord injury in rats," Spinal Cord, vol. 44, no. 11, pp. 662-667, 2006.

[130] J. Barnes, L. A. Anderson, and J. D. Phillipson, “St John's wort (Hypericum perforatum L.): a review of its chemistry, 
pharmacology and clinical properties," The Journal of Pharmacy and Pharmacology, vol. 53, no. 5, pp. 583-600, 2001.

[131] J. M. Greeson, B. Sanford, and D. A. Monti, “St. John's wort (Hypericum perforatum): a review of the current pharmacological, toxicological, and clinical literature," Psychopharmacology, vol. 153, no. 4, pp. 402-414, 2001.

[132] J. Patočka, “The chemistry, pharmacology, and toxicology of the biologically active constituents of the herb Hypericum perforatum L," Journal of Applied Biomedicine, vol. 1, no. 2, pp. 61-70, 2003.

[133] M. Nakayama, M. Aihara, Y. N. Chen, M. Araie, K. TomitaYokotani, and T. Iwashina, "Neuroprotective effects of flavonoids on hypoxia-, glutamate-, and oxidative stress-induced retinal ganglion cell death," Molecular Vision, vol. 17, pp. 1784-1793, 2011.

[134] K. E. Heim, A. R. Tagliaferro, and D. J. Bobilya, "Flavonoid antioxidants: chemistry, metabolism and structure-activity relationships," The Journal of Nutritional Biochemistry, vol. 13, no. 10, pp. 572-584, 2002.

[135] O. Krishtal, N. Lozovaya, A. Fisunov et al., "Modulation of ion channels in rat neurons by the constituents of Hypericum perforatum," Pharmacopsychiatry, vol. 34, Supplement 1, pp. 74-82, 2001.

[136] V. Kumar, A. Mdzinarishvili, C. Kiewert et al., "NMDA receptor-antagonistic properties of hyperforin, a constituent of St John's Wort," Journal of Pharmacological Sciences, vol. 102, no. 1, pp. 47-54, 2006.

[137] K. M. Vance, D. M. Ribnicky, G. E. Hermann, and R. C. Rogers, "St. John's Wort enhances the synaptic activity of the nucleus of the solitary tract," Nutrition, vol. 30, 7-8 Supplement, pp. S37-S42, 2014.

[138] J. Benedi, R. Arroyo, C. Romero, S. Martín-Aragón, and A. M. Villar, "Antioxidant properties and protective effects of a standardized extract of Hypericum perforatum on hydrogen peroxide-induced oxidative damage in PC12 cells," Life Sciences, vol. 75, no. 10, pp. 1263-1276, 2004.

[139] Ü. S. Özdemir, M. Nazıroğlu, N. Şenol, and V. Ghazizadeh, "Hypericum perforatum attenuates spinal cord injuryinduced oxidative stress and apoptosis in the dorsal root ganglion of rats: involvement of TRPM2 and TRPV1 channels," Molecular Neurobiology, vol. 53, no. 6, pp. 35403551, 2016.

[140] N. Osakabe, A. Yasuda, M. Natsume et al., "Rosmarinic acid, a major polyphenolic component of Perilla frutescens, reduces lipopolysaccharide (LPS)-induced liver injury in Dgalactosamine (D-GalN)-sensitized mice," Free Radical Biology \& Medicine, vol. 33, no. 6, pp. 798-806, 2002.

[141] W. Boonyarikpunchai, S. Sukrong, and P. Towiwat, "Antinociceptive and anti-inflammatory effects of rosmarinic acid isolated from Thunbergia laurifolia Lindl.," Pharmacology, Biochemistry, and Behavior, vol. 124, pp. 67-73, 2014.

[142] M. Petersen, "Rosmarinic acid: new aspects," Phytochemistry Reviews, vol. 12, no. 1, pp. 207-227, 2013.

[143] A. J. Shang, Y. Yang, H. Y. Wang et al., "Spinal cord injury effectively ameliorated by neuroprotective effects of rosmarinic acid," Nutritional Neuroscience, vol. 20, no. 3, pp. 172179, 2017.

[144] Z. H. Wang, Y. X. Xie, J. W. Zhang et al., "Carnosol protects against spinal cord injury through Nrf-2 upregulation," Journal of Receptor and Signal Transduction Research, vol. 36, no. 1, pp. 72-78, 2016.
[145] M. C. Comelli, U. Mengs, C. Schneider, and M. Prosdocimi, "Toward the definition of the mechanism of action of silymarin: activities related to cellular protection from toxic damage induced by chemotherapy," Integrative Cancer Therapies, vol. 6, no. 2, pp. 120-129, 2007.

[146] P. F. Surai, "Silymarin as a natural antioxidant: an overview of the current evidence and perspectives," Antioxidants, vol. 4, no. 1, pp. 204-247, 2015.

[147] M. J. Tsai, J. F. Liao, D. Y. Lin et al., "Silymarin protects spinal cord and cortical cells against oxidative stress and lipopolysaccharide stimulation," Neurochemistry International, vol. 57, no. 8, pp. 867-875, 2010.

[148] A. Borah, R. Paul, S. Choudhury et al., "Neuroprotective potential of silymarin against CNS disorders: insight into the pathways and molecular mechanisms of action," CNS Neuroscience \& Therapeutics, vol. 19, no. 11, pp. 847-853, 2013.

[149] T. G. Son, S. Camandola, T. V. Arumugam et al., "Plumbagin, a novel Nrf2/ARE activator, protects against cerebral ischemia," Journal of Neurochemistry, vol. 112, no. 5, pp. 13161326, 2010.

[150] W. Zhang, L. Cheng, Y. Hou, M. Si, Y. P. Zhao, and L. Nie, "Plumbagin protects against spinal cord injury-induced oxidative stress and inflammation in Wistar rats through Nrf-2 upregulation," Drug Res (Stuttg)., vol. 65, no. 9, pp. 495499, 2015.

[151] W. Li, T. O. Khor, C. Xu et al., "Activation of Nrf2antioxidant signaling attenuates $\mathrm{NF} \kappa \mathrm{B}$-inflammatory response and elicits apoptosis," Biochemical Pharmacology, vol. 76, no. 11, pp. 1485-1489, 2008.

[152] C. Shi, S. Ahmad Khan, K. Wang, and M. Schneider, "Improved delivery of the natural anticancer drug tetrandrine," International Journal of Pharmaceutics, vol. 479, no. 1, pp. 41-51, 2015.

[153] Y. J. Chen, "Potential role of tetrandrine in cancer therapy," Acta Pharmacologica Sinica, vol. 23, no. 12, pp. 1102-1106, 2002.

[154] S. J. Wu and L. T. Ng, "Tetrandrine inhibits proinflammatory cytokines, iNOS and COX-2 expression in human monocytic cells," Biological \& Pharmaceutical Bulletin, vol. 30, no. 1, pp. 59-62, 2007.

[155] S. J. Liu, S. W. Zhou, and C. S. Xue, "Effect of tetrandrine on neutrophilic recruitment response to brain ischemia/reperfusion," Acta Pharmacologica Sinica, vol. 22, no. 11, pp. 971975, 2001.

[156] Y. Chen, Y. H. Tsai, and S. H. Tseng, "The potential of tetrandrine as a protective agent for ischemic stroke," Molecules, vol. 16, no. 9, pp. 8020-8032, 2011.

[157] G. Bao, C. Li, L. Qi, N. Wang, and B. He, "Tetrandrine protects against oxygen-glucose-serum deprivation/reoxygenation- induced injury via $\mathrm{PI} 3 \mathrm{~K} / \mathrm{AKT} / \mathrm{NF}-\kappa \mathrm{B}$ signaling pathway in rat spinal cord astrocytes," Biomedicine \& Pharmacotherapy, vol. 84, pp. 925-930, 2016.

[158] X. Xia, Y. Ma, L. B. Yang et al., "Impact of heat shock protein A $12 \mathrm{~B}$ overexpression on spinal astrocyte survival against oxygen-glucose-serum deprivation/restoration in primary cultured astrocytes," Journal of Molecular Neuroscience, vol. 59, no. 4, pp. 511-520, 2016.

[159] F. Tian, L. H. Xu, W. Zhao, L. J. Tian, and X. L. Ji, “The optimal therapeutic timing and mechanism of puerarin treatment of spinal cord ischemia-reperfusion injury in rats," Journal of Ethnopharmacology, vol. 134, no. 3, pp. 892-896, 2011. 
[160] F. Tian, L. H. Xu, W. Zhao, L. J. Tian, and X. L. Ji, "The neuroprotective mechanism of puerarin treatment of acute spinal cord injury in rats," Neuroscience Letters, vol. 543, pp. 64-68, 2013.

[161] F. Tian, L. H. Xu, B. Wang, L. J. Tian, and X. L. Ji, “The neuroprotective mechanism of puerarin in the treatment of acute spinal ischemia-reperfusion injury is linked to cyclindependent kinase 5," Neuroscience Letters, vol. 584, pp. 50$55,2015$.

[162] D. Zhang, G. Ma, M. Hou, T. Zhang, L. Chen, and C. Zhao, "The neuroprotective effect of puerarin in acute spinal cord injury rats," Cellular Physiology and Biochemistry, vol. 39, no. 3, pp. 1152-1164, 2016.

[163] M. Wu, G. Zhao, X. Yang et al., "Puerarin accelerates neural regeneration after sciatic nerve injury," Neural Regeneration Research, vol. 9, no. 6, pp. 589-593, 2014.

[164] M. Liu, K. Liao, C. Yu, X. Li, S. Liu, and S. Yang, "Puerarin alleviates neuropathic pain by inhibiting neuroinflammation in spinal cord," Mediators of Inflammation, vol. 2014, Article ID 485927, 9 pages, 2014.

[165] L. Bayan, P. H. Koulivand, and A. Gorji, "Garlic: a review of potential therapeutic effects," Avicenna Journal of Phytomedicine, vol. 4, no. 1, pp. 1-14, 2014.

[166] K. Alam, O. Hoq, and S. Uddin, "Medicinal plant Allium sativum. A review," Journal of Medicinal Plant Studies, vol. 4, no. 6, pp. 72-79, 2016.

[167] S. G. Liu, P. Y. Ren, G. Y. Wang, S. X. Yao, and X. J. He, “Allicin protects spinal cord neurons from glutamate-induced oxidative stress through regulating the heat shock protein 70/inducible nitric oxide synthase pathway," Food \& Function, vol. 6, no. 1, pp. 320-329, 2015.

[168] L. D. Lawson and C. D. Gardner, "Composition, stability, and bioavailability of garlic products used in a clinical trial," Journal of Agricultural and Food Chemistry, vol. 53, no. 16, pp. 6254-6261, 2005.

[169] W. Huang, Y. Wang, Y. G. Cao et al., “Antiarrhythmic effects and ionic mechanisms of allicin on myocardial injury of diabetic rats induced by streptozotocin," Naunyn-Schmiedeberg's Archives of Pharmacology, vol. 386, no. 8, pp. 697704, 2013.

[170] S. Wang and D. Ren, "Allicin protects traumatic spinal cord injury through regulating the HSP70/Akt/iNOS pathway in mice," Molecular Medicine Reports, vol. 14, no. 4, pp. 30863092, 2016.

[171] S. C. Gupta, S. Prasad, J. H. Kim et al., "Multitargeting by curcumin as revealed by molecular interaction studies," Natural Product Reports, vol. 28, no. 12, pp. 1937-1955, 2011.

[172] P. Maiti and G. L. Dunbar, "Use of curcumin, a natural polyphenol for targeting molecular pathways in treating agerelated neurodegenerative diseases," International Journal of Molecular Sciences, vol. 19, no. 6, p. 1637, 2018.

[173] A. Bhat, A. M. Mahalakshmi, B. Ray et al., "Benefits of curcumin in brain disorders," BioFactors, vol. 45 , no. 5, pp. 666689, 2019.

[174] M. S. Lin, Y. H. Lee, W. T. Chiu, and K. S. Hung, "Curcumin provides neuroprotection after spinal cord injury," The Journal of Surgical Research, vol. 166, no. 2, pp. 280-289, 2011.

[175] L. G. Costa, J. M. Garrick, P. J. Roquè, and C. Pellacani, "Mechanisms of neuroprotection by quercetin: counteracting oxidative stress and more," Oxidative Medicine and Cellular Longevity, vol. 2016, Article ID 2986796, 10 pages, 2016.
[176] D. Del Rio, A. Rodriguez-Mateos, J. P. Spencer, M. Tognolini, G. Borges, and A. Crozier, "Dietary (poly)phenolics in human health: structures, bioavailability, and evidence of protective effects against chronic diseases," Antioxidants \& Redox Signaling, vol. 18, no. 14, pp. 1818-1892, 2013.

[177] A. W. Boots, G. R. Haenen, and A. Bast, "Health effects of quercetin: from antioxidant to nutraceutical," European Journal of Pharmacology, vol. 585, no. 2-3, pp. 325-337, 2008.

[178] B. Halliwell, J. Rafter, and A. Jenner, "Health promotion by flavonoids, tocopherols, tocotrienols, and other phenols: direct or indirect effects? Antioxidant or not?," The American Journal of Clinical Nutrition, vol. 81, no. 1, pp. 268S-276S, 2005.

[179] C. G. Fraga, M. Galleano, S. V. Verstraeten, and P. I. Oteiza, "Basic biochemical mechanisms behind the health benefits of polyphenols," Molecular Aspects of Medicine, vol. 31, no. 6, pp. 435-445, 2010.

[180] G. S. Kelly, "Quercetin. Monograph," Alternative Medicine Review : A Journal of Clinical Therapeutic, vol. 16, no. 2, pp. 172-194, 2011.

[181] M. Russo, C. Spagnuolo, I. Tedesco, S. Bilotto, and G. L. Russo, "The flavonoid quercetin in disease prevention and therapy: facts and fancies," Biochemical Pharmacology, vol. 83, no. 1, pp. 6-15, 2012.

[182] B. Ossola, T. M. Kääriäinen, and P. T. Männistö, “The multiple faces of quercetin in neuroprotection," Expert Opinion on Drug Safety, vol. 8, no. 4, pp. 397-409, 2009.

[183] A. Ishisaka, S. Ichikawa, H. Sakakibara et al., "Accumulation of orally administered quercetin in brain tissue and its antioxidative effects in rats," Free Radical Biology \& Medicine, vol. 51, no. 7, pp. 1329-1336, 2011.

[184] S. Das, A. K. Mandal, A. Ghosh, S. Panda, N. Das, and S. Sarkar, "Nanoparticulated quercetin in combating age related cerebral oxidative injury," Current Aging Science, vol. 1, no. 3, pp. 169-174, 2008.

[185] C. Unsal, M. Kanter, C. Aktas, and M. Erboga, "Role of quercetin in cadmium-induced oxidative stress, neuronal damage, and apoptosis in rats," Toxicology and Industrial Health, vol. 31, no. 12, pp. 1106-1115, 2015.

[186] Y. Zhang, B. Yi, J. Ma et al., "Quercetin promotes neuronal and behavioral recovery by suppressing inflammatory response and apoptosis in a rat model of intracerebral hemorrhage," Neurochemical Research, vol. 40, no. 1, pp. 195-203, 2015.

[187] E. Schültke, E. Kendall, H. Kamencic, Z. Ghong, R. W. Griebel, and B. H. Juurlink, "Quercetin promotes functional recovery following acute spinal cord injury," Journal of $\mathrm{Neu}$ rotrauma, vol. 20, no. 6, pp. 583-591, 2003.

[188] Y. Wang, W. Li, M. Wang et al., "Quercetin reduces neural tissue damage and promotes astrocyte activation after spinal cord injury in rats," Journal of Cellular Biochemistry, vol. 119, no. 2, pp. 2298-2306, 2018.

[189] M. A. Alam, V. P. Subramanyam Rallabandi, and P. K. Roy, "Systems biology of immunomodulation for post-stroke neuroplasticity: multimodal implications of pharmacotherapy and neurorehabilitation," Frontiers in Neurology, vol. 7, p. 94, 2016.

[190] A. Tabarroki and R. V. Tiu, "Molecular genetics of myelofibrosis and its associated disease phenotypes," Translational medicine@UniSa, vol. 8,pp. 53-64, 2014. 
[191] A. A. Qureshi, H. Mo, L. Packer, and D. M. Peterson, "Isolation and identification of novel tocotrienols from rice bran with hypocholesterolemic, antioxidant, and antitumor properties," Journal of Agricultural and Food Chemistry, vol. 48, no. 8, pp. 3130-3140, 2000.

[192] H. Ahsan, A. Ahad, J. Iqbal, and W. A. Siddiqui, "Pharmacological potential of tocotrienols: a review," Nutrition \& Metabolism, vol. 11, no. 1, p. 52, 2014.

[193] C. K. Sen, S. Khanna, and S. Roy, "Tocotrienols: vitamin E beyond tocopherols," Life Sciences, vol. 78, no. 18, pp. 2088-2098, 2006.

[194] K. Hensley, E. J. Benaksas, R. Bolli et al., "New perspectives on vitamin E: $\gamma$-tocopherol and carboxyethylhydroxychroman metabolites in biology and medicine," Free Radical Biology \& Medicine, vol. 36, no. 1, pp. 1-15, 2004.

[195] E. H. Siemann and L. L. Creasy, "Concentration of the phytoalexin resveratrol in wine," American Journal of Enology and Viticulture, vol. 43, pp. 49-52, 1992.

[196] S. D. Rege, S. Kumar, D. N. Wilson et al., "Resveratrol protects the brain of obese mice from oxidative damage," Oxidative Medicine and Cellular Longevity, vol. 2013, Article ID 419092, 7 pages, 2013.

[197] K. Sinha, G. Chaudhary, and Y. K. Gupta, "Protective effect of resveratrol against oxidative stress in middle cerebral artery occlusion model of stroke in rats," Life Sciences, vol. 71, no. 6, pp. 655-665, 2002.

[198] G. Cheng, X. Zhang, D. Gao, X. Jiang, and W. Dong, "Resveratrol inhibits MMP-9 expression by up-regulating PPAR $\alpha$ expression in an oxygen glucose deprivation-exposed neuron model," Neuroscience Letters, vol. 451, no. 2, pp. 105-108, 2009.

[199] H. Zhuang, Y. S. Kim, R. C. Koehler, and S. Doré, "Potential mechanism by which resveratrol, a red wine constituent, protects neurons," Annals of the New York Academy of Sciences, vol. 993, no. 1, pp. 276-286, 2003.

[200] J. Yang, J. Huang, C. Shen et al., "Resveratrol treatment in different time-attenuated neuronal apoptosis after oxygen and glucose deprivation/reoxygenation via enhancing the activation of Nrf-2 signaling pathway in vitro," Cell Transplantation, vol. 27, no. 12, pp. 1789-1797, 2018.

[201] M. Virgili and A. Contestabile, "Partial neuroprotection of in vivo excitotoxic brain damage by chronic administration of the red wine antioxidant agent, trans-resveratrol in rats," Neuroscience Letters, vol. 281, no. 2-3, pp. 123-126, 2000.

[202] C. Girbovan, L. Morin, and H. Plamondon, "Repeated resveratrol administration confers lasting protection against neuronal damage but induces dose-related alterations of behavioral impairments after global ischemia," Behavioural Pharmacology, vol. 23, no. 1, pp. 1-13, 2012.

[203] S. S. Huang, M. C. Tsai, C. L. Chih, L. M. Hung, and S. K. Tsai, "Resveratrol reduction of infarct size in Long-Evans rats subjected to focal cerebral ischemia," Life Sciences, vol. 69, no. 9, pp. 1057-1065, 2001.

[204] F. Karalis, V. Soubasi, T. Georgiou et al., "Resveratrol ameliorates hypoxia/ischemia-induced behavioral deficits and brain injury in the neonatal rat brain," Brain Research, vol. 1425, pp. 98-110, 2011.

[205] R. H. Singleton, H. Q. Yan, W. Fellows-Mayle, and C. E. Dixon, "Resveratrol attenuates behavioral impairments and reduces cortical and hippocampal loss in a rat controlled cor- tical impact model of traumatic brain injury," Journal of Neurotrauma, vol. 27, no. 6, pp. 1091-1099, 2010.

[206] U. Sönmez, A. Sönmez, G. Erbil, I. Tekmen, and B. Baykara, "Neuroprotective effects of resveratrol against traumatic brain injury in immature rats," Neuroscience Letters, vol. 420, no. 2, pp. 133-137, 2007.

[207] A. W. Shao, H. J. Wu, S. Chen, A. B. Ammar, J. M. Zhang, and Y. Hong, "Resveratrol Attenuates Early Brain Injury After Subarachnoid Hemorrhage Through Inhibition of NF- $\kappa$ BDependent Inflammatory/MMP-9 Pathway," CNS Neuroscience \& Therapeutics, vol. 20, no. 2, pp. 182-185, 2014.

[208] O. Ates, S. Cayli, E. Altinoz et al., "Effects of resveratrol and methylprednisolone on biochemical, neurobehavioral and histopathological recovery after experimental spinal cord injury," Acta Pharmacologica Sinica, vol. 27, no. 10, pp. 1317-1325, 2006.

[209] S. Kaplan, G. Bisleri, J. A. Morgan, F. H. Cheema, and M. C. $\mathrm{Oz}$, "Resveratrol, a Natural Red Wine Polyphenol, Reduces Ischemia-Reperfusion- Induced Spinal Cord Injury," The Annals of Thoracic Surgery, vol. 80, no. 6, pp. 2242-2249, 2005.

[210] C. Liu, Z. Shi, L. Fan, C. Zhang, K. Wang, and B. Wang, "Resveratrol improves neuron protection and functional recovery in rat model of spinal cord injury," Brain Research, vol. 1374, pp. 100-109, 2011.

[211] M. Takanashi, 1998, U.S. Patent 5,773,004.

[212] C. C. Lin, M. L. Lin, and J. M. Lin, "The antiinflammatory and liver protective effect of Tithonia diversifolia (Hemsl.) gray and Dicliptera chinensis Juss. Extracts in rats," Phytotherapy Research, vol. 7, no. 4, pp. 305-309, 1993.

[213] A. Schuster, S. Stokes, F. Papastergiou, V. Castro, L. Poveda, and J. Jakupovic, "Sesquiterpene lactones from two Tithonia species," Phytochemistry, vol. 31, no. 9, pp. 3139-3141, 1992.

[214] M. Bordoloi, N. C. Barua, and A. C. Ghosh, "An artemisinic acid analogue from Tithonia diversifolia," Phytochemistry, vol. 41, no. 2, pp. 557-559, 1996.

[215] Y. H. Kuo and C. H. Chen, "Sesquiterpenes from the leaves of Tithonia diversifolia," Journal of Natural Products, vol. 61, no. 6, pp. 827-828, 1998.

[216] C. L. Juang, F. S. Yang, M. S. Hsieh, H. Y. Tseng, S. C. Chen, and H. C. Wen, "Investigation of anti-oxidative stress in vitro and water apparent diffusion coefficient in MRI on rat after spinal cord injury in vivo with Tithonia diversifolia ethanolic extracts treatment," BMC Complementary and Alternative Medicine, vol. 14, no. 1, p. 447, 2014.

[217] L. Wei and L. Zhang, "Effects of Danshen injection on glial cell line-derived neurotrophic factor mRNA of acute spinal cord injury rats and its mechanisms," Zhongguo Zhong Xi Yi Jie He Za Zhi, vol. 33, no. 7, pp. 933-937, 2013.

[218] Y. G. Yu, J. Yang, X. H. Cheng et al., "The protection of acute spinal cord injury by subarachnoid space injection of Danshen in animal models," The Journal of Spinal Cord Medicine, vol. 42, no. 3, pp. 355-359, 2019.

[219] Z. K. Fan, G. Lv, Y. F. Wang et al., "The protective effect of salvianolic acid B on blood-spinal cord barrier after compression spinal cord injury in rats," Journal of Molecular Neuroscience, vol. 51, no. 3, pp. 986-993, 2013.

[220] D. S. Yu, Y. S. Wang, Y. L. Bi et al., "Salvianolic acid A ameliorates the integrity of blood-spinal cord barrier via miR101/Cul3/Nrf2/HO-1 signaling pathway," Brain Research, vol. 1657, pp. 279-287, 2017. 
[221] Z. Zhu, L. Ding, W. F. Qiu, H. F. Wu, and R. Li, "Salvianolic acid B protects the myelin sheath around injured spinal cord axons," Neural Regeneration Research, vol. 11, no. 3, pp. 487492, 2016.

[222] X. Chen, C. Zhou, J. Guo et al., "Effects of dihydroxylphenyl lactic acid on inflammatory responses in spinal cord injury," Brain Research, vol. 1372, pp. 160-168, 2011.

[223] X. Yin, Y. Yin, F. L. Cao et al., “Tanshinone IIA attenuates the inflammatory response and apoptosis after traumatic injury of the spinal cord in adult rats," PLoS One, vol. 7, no. 6, article e38381, 2012.

[224] L. Zhang, W. Gan, and G. An, "Influence of tanshinone IIa on heat shock protein 70, Bcl-2 and Bax expression in rats with spinal ischemia/reperfusion injury," Neural Regeneration Research, vol. 7, no. 36, pp. 2882-2888, 2012.

[225] X. M. Zhang, J. Ma, Y. Sun et al., “Tanshinone IIA promotes the differentiation of bone marrow mesenchymal stem cells into neuronal-like cells in a spinal cord injury model," Journal of Translational Medicine, vol. 16, no. 1, p. 193, 2018.

[226] J. Tang, C. Zhu, Z. H. Li et al., "Inhibition of the spinal astrocytic JNK/MCP-1 pathway activation correlates with the analgesic effects of tanshinone IIA sulfonate in neuropathic pain," Journal of Neuroinflammation, vol. 12, p. 57, 2015.

[227] Y. D. Yang, X. Yu, X. M. Wang, X. H. Mu, and F. He, “Tanshinone IIA improves functional recovery in spinal cord injury-induced lower urinary tract dysfunction," Neural Regeneration Research, vol. 12, no. 2, pp. 267-275, 2017. 